\title{
Regularity results for pluriclosed flow
}

\author{
JEFFREY STREETS \\ GANG TIAN
}

\begin{abstract}
In [27] the authors introduced a parabolic flow of pluriclosed metrics. Here we give improved regularity results for solutions to this equation. Furthermore, we exhibit this equation as the gradient flow of the lowest eigenvalue of a certain Schrödinger operator, and show the existence of an expanding entropy functional for this flow. Finally, we motivate a conjectural picture of the optimal regularity results for this flow, and discuss some of the consequences.
\end{abstract}

$32 \mathrm{Q} 55,53 \mathrm{C} 44,53 \mathrm{C} 55$

\section{Introduction}

Let $\left(M^{2 n}, J\right)$ be a complex manifold, and let $\omega$ denote a Hermitian metric on $M$. The metric $\omega$ is pluriclosed if

$$
\partial \bar{\partial} \omega=0 .
$$

Consider the initial value problem:

$$
\begin{aligned}
\frac{\partial}{\partial t} \omega & =\partial \partial^{*} \omega+\bar{\partial} \bar{\partial}^{*} \omega+\frac{\sqrt{-1}}{2} \partial \bar{\partial} \log \operatorname{det} g, \\
\omega(0) & =\omega_{0} .
\end{aligned}
$$

This equation was introduced in Streets and Tian [27] as a tool for understanding complex, non-Kähler manifolds. Equation (1-1) falls into a general class of flows of Hermitian metrics, and as shown in Streets and Tian [28], solutions to (1-1) exist as long as the Chern curvature, torsion and covariant derivative of torsion are bounded. This is analogous to the long-time existence theorem by $\mathrm{R}$ Hamilton [14, Theorem 14.1], which states that the Ricci flow with any initial data has a solution on $[0, T)$, where either $T=\infty$ or the curvature of the solution blows up at time $T$. A natural problem is whether or not we can drop the hypothesis that the torsion and its first covariant derivative is bounded at a finite singular time for (1-1). In the case $n=2$, we showed in [27] that a bound on the Chern curvature suffices to show long-time existence. The difficulty in general arises from the fact that the induced evolution equation on the Chern curvature involves the torsion and its derivatives. Our crucial observation 
for overcoming this difficulty is that the Bismut connection is a much more natural connection for studying (1-1).

In this paper, we will give sharper long-time existence theorems for (1-1). We will also prove some useful regularity theorems and paint a more concrete picture for the conjectural existence and singularity formation for the flow (1-1). Furthermore, by giving an interesting interpretation of the flow using the Bismut connection, we exhibit a remarkable relationship of (1-1) to mathematical physics. Specifically, we show that up to gauge equivalence (1-1) is the renormalization group flow of a nonlinear sigma model with nonzero $B$-field. As a consequence we derive that (1-1) is a gradient flow, and exhibit a certain entropy functional. Finally, we discuss some applications of our conjectural picture to understanding the topology of Class VII surfaces.

We start by recalling the Bismut connection. Let $\left(M^{2 n}, \omega, J\right)$ be a complex manifold with pluriclosed metric. Let $D$ denote the Levi-Civita connection. Then the Bismut connection $\nabla$ is defined via

$$
\left\langle\nabla_{X} Y, Z\right\rangle=\left\langle D_{X} Y, Z\right\rangle+\frac{1}{2} d^{c} \omega(X, Y, Z),
$$

where $d^{c} \omega(X, Y, Z):=d \omega(J X, J Y, J Z)$. Let $\Omega$ denote the curvature of this connection, and let $P$ denote the Chern form of this connection, ie, in complex coordinates:

$$
P_{i \bar{j}}=\Omega_{i \bar{j} k}^{k}
$$

Finally, let $P^{C}$ denote the Ricci form associated to the Chern connection. One can calculate (Alexandrov and Ivanov [1]) that

$$
P=P^{C}-d d^{*} \omega \text {. }
$$

In particular, this implies that a solution to (1-1) may be expressed as

$$
\frac{\partial}{\partial t} \omega=-P^{1,1}
$$

where $P^{1,1}$ denotes the projection of $P$ onto $(1,1)$-forms. This is a convenient framework for understanding solutions to (1-1). In particular, with the clarifying lens of this connection, we are able to show that (1-1) is the gradient flow of the first eigenvalue of a particular Schrödinger operator. First generalize the notation slightly and let $\left(M^{n}, g\right)$ be a Riemannian manifold, and let $T$ denote a three-form on $M$. Let

$$
\mathcal{F}(g, T, f)=\int_{M}\left[R-\frac{1}{12}|T|^{2}+|\nabla f|^{2}\right] e^{-f} d V .
$$

Furthermore set

$$
\lambda(g, T)=\inf _{\left\{f \mid \int_{M} e^{-f} d V=1\right\}} F(g, T, f) .
$$


In Section 6 we exhibit Equation (1-1) as the gradient flow of $\lambda$.

Theorem 1.1 Let $\left(M^{2 n}, \omega, J\right)$ be a complex manifold with pluriclosed metric. Let $\omega(t)$ denote the solution to (1-1) with initial condition $\omega$, and let $g(t)$ be the associated metric, and $T(t)$ the torsions of the associated Bismut connections. Let Met denote the space of smooth metrics on $M$, and let

$$
\mathcal{M}:=\frac{\left\{(g, T) \mid g \in \operatorname{Met}, T \in \Lambda^{3}, d T=0\right\}}{\operatorname{Diff}_{+}(M)},
$$

where Diff + is the group of oriented diffeomorphisms of $M$, acting naturally on $g$ and $T$. The pair $(g(t), T(t))$ is a solution of the gradient flow of $\lambda$ acting on $\mathcal{M}$.

More specifically, we show that after pulling back by the one-parameter family of diffeomorphisms generated by the Lee forms of the time dependent metrics, Equation (1-1) is unmasked as the $B$-field renormalization group flow of string theory. This flow has been previously studied, and admits the generalization $\lambda$ of the Perelman energy (Oliynyk, Suneeta and Woolgar [21]). Furthermore an expanding entropy functional for this flow was discovered by the first named author [26], which hence is monotone for solutions to (1-1) as well. These observations show that any breather solution is automatically a gradient soliton (Corollary 6.11), and furthermore imply strong results on certain long-time solutions.

Turning to the regularity theory, we show that a bound on the Bismut Ricci curvature suffices to obtain long-time existence for solutions of (1-1).

Theorem 1.2 Let $\left(M^{2 n}, \omega(t), J\right)$ be a solution to (1-1) on $[0, \tau)$. Suppose

$$
\int_{0}^{\tau} \sup _{M \times\{t\}}\left|P^{1,1}\right| d t<\infty
$$

Then the solution extends smoothly past time $\tau$.

This theorem is analogous to a result of N Sesum for the Ricci flow [25, Theorem 2], and already represents a significant improvement, as we have reduced the regularity requirement to understanding the Ricci-type curvature of a specific connection.

The theory of Kähler Ricci flow is considerably more developed than the general study of Ricci flow. One of key reasons for this is the reduction of the Kähler Ricci flow to a scalar equation. Inspired by this, we will introduce a certain potential function $\phi$ along a solution to (1-1) and prove a regularity theorem in term of this potential and 
the torsion. Let $\left(M^{2 n}, \widetilde{\omega}, J\right)$ be a complex manifold with pluriclosed metric, and let $\omega(t)$ be a solution to (1-1). We define

$$
\begin{aligned}
\frac{\partial}{\partial t} \phi-\Delta \phi & =\operatorname{tr}_{\omega} \widetilde{\omega}-n, \\
\phi(0) & =0 .
\end{aligned}
$$

Here $\Delta$ is the canonical Laplacian associated to the time dependent metric $\omega(t)$, ie, $\Delta=\operatorname{tr}_{\omega} \partial \bar{\partial}$. It follows from standard parabolic theory that $\phi$ exists on the same time interval that $\omega(t)$ exists. More generally, one may define this with respect to a one-parameter family of background metrics $\widetilde{\omega}(t)$. Related quantities for MongeAmpère-type equations on almost Kähler surfaces were considered in Weinkove [33].

Theorem 1.3 Let $\left(M^{2 n}, \tilde{g}, J\right)$ be a compact complex manifold and suppose $g(t)$ is a solution to (1-1) on $[0, \tau)$ and suppose there is a constant $C$ such that:

$$
\sup _{M \times[0, \tau)}|\phi| \leq C, \quad \sup _{M \times[0, \tau)}|T|^{2} \leq C .
$$

Then $g(t) \rightarrow g(\tau)$ in $C^{\infty}$, and the flow extends smoothly past time $\tau$.

This represents a significant reduction of the regularity requirement for solutions to (1-1), effectively reducing the question to understanding the behavior of the potential function and the torsion. The proof involves applying the maximum principle to carefully chosen quantities. Going further, one would like to understand what the optimal existence and regularity theorems are for (1-1). For this we again take a cue from the study of Kähler Ricci flow. Suppose $\left(M^{2 n}, \omega_{0}, J\right)$ is a Kähler manifold, and recall the Kähler Ricci flow equation:

$$
\begin{aligned}
\frac{\partial}{\partial t} \omega & =\frac{\sqrt{-1}}{2} \partial \bar{\partial} \log \operatorname{det} g, \\
\omega(0) & =0 .
\end{aligned}
$$

Associated to a solution $\omega(t)$ of (1-4) is an $\operatorname{ODE}$ in $H^{2}(M, \mathbb{R})$, which has solution:

$$
[\omega(t)]=\left[\omega_{0}\right]-t c_{1}(M) .
$$

The optimal regularity theorems for Kähler Ricci flow assert that as long as the solution to the ODE above remains in the Kähler cone, the solution exists up to that time (Tian and Zhang [32]; see also Tian [31]). An essential ingredient of these theorems is the reduction of Kähler Ricci flow to a scalar equation, then exploiting the estimates of the Monge-Ampère equation using the maximum principle. 
Keeping with this theme, observe that a pluriclosed metric defines a class in the Aeppli cohomology group:

$$
\mathcal{H}_{\partial+\bar{\partial}}^{1,1}=\frac{\left\{\operatorname{Ker} \partial \bar{\partial}: \Lambda_{\mathbb{R}}^{1,1} \rightarrow \Lambda_{\mathbb{R}}^{2,2}\right\}}{\left\{\partial \alpha+\bar{\partial} \bar{\alpha} \mid \alpha \in \Lambda^{0,1}\right\}} .
$$

Define the space $\mathcal{P}_{\partial+\bar{\partial}}$ to be the cone of the classes in $\mathcal{H}_{\partial+\bar{\partial}}$ that contain positivedefinite elements. Solutions to Equation (1-1) clearly define ODEs in $\mathcal{H}_{\partial+\bar{\partial}}^{1,1}$, and it is natural to conjecture (see Conjecture 5.2) that the maximal existence time is characterized by the first time at which the boundary of $\mathcal{P}_{\partial+\bar{\partial}}$ is reached. If true, this would have strong implications on complex surfaces since the cone $\mathcal{P}_{\partial+\bar{\partial}}$ is essentially characterized on complex surfaces in terms of the action of the class in $\mathcal{H}_{\partial+\bar{\partial}}$ on curves. More precisely, if $\phi$ is a pluriclosed (1,1)-form on a complex non-Kähler surface $\left(M^{4}, J\right)$, then $\phi \in \mathcal{P}_{\partial+\bar{\partial}}$ if and only if (1) $\int_{M} \phi \wedge \gamma_{0}>0$, (2) $\int_{D} \phi>0$ for every effective divisor with negative self-intersection. Here $\gamma_{0}$ is the kernel of the projection map from the $(1,1)$ Bott-Chern cohomology of $M$ to $H^{1,1}$, explained further in Section 5.

To further illustrate the significance of the cone $\mathcal{P}_{\partial+\bar{\partial}}$, we show in Section 5 that as long as the solution to the associated ODE remains in the interior of $\mathcal{P}$, solutions to (1-1) on complex surfaces may be canonically reduced to solutions of a certain PDE on $\alpha \in \Lambda^{0,1}$, and an ODE on $\psi \in \Lambda^{1,1}$. Specifically, we can find a background metric $\tilde{g}$ so that, setting $\omega(t)=\omega(0)+\partial \alpha+\bar{\partial} \bar{\alpha}+\psi$, one has the solution to (1-1) reduced to:

$$
\begin{aligned}
\frac{\partial}{\partial t} \alpha & =\partial_{\omega}^{*} \omega+\frac{\sqrt{-1}}{4} \bar{\partial} \log \frac{\omega^{n}}{\widetilde{\omega}^{n}}, \\
\frac{\partial}{\partial t} \psi & =-c_{1}(\widetilde{g}), \\
\alpha(0) & =\alpha_{0}, \quad \psi(0)=\psi_{0} .
\end{aligned}
$$

This equation may be taken as an ansatz for Equation (1-1) in any dimension. We discuss some further properties of this equation in Section 5.

Finally, in Section 7 we examine nonsingular solutions to (1-1), suitably normalized, on Class $\mathrm{VII}^{+}$surfaces. By exploiting certain monotone quantities we show that a positive resolution of the conjectural picture of singularity formation outlined in Section 5 implies the existence of a curve on a Class VII ${ }^{+}$surface. Due to the results of Nakamura [20], and Dloussky, Oeljeklaus and Toma [9], the classification of such surfaces reduces to finding sufficiently many curves. In particular we show that our conjectural picture implies the classification of Class $\mathrm{VII}^{+}$surfaces with $b_{2}=1$. This is discussed further in Section 7.

Here is an outline of the rest of the paper. In Section 2 we establish certain differential inequalities for solutions to (1-1), which are inspired by the theory of the complex 
Monge-Ampère equation, which can be used to establish uniform bounds on the metric. Next in Section 3 we derive a $C^{1}$ estimate for the metric along solutions to (1-1) under certain hypotheses. Building on these estimates, in Section 4 we give the proofs of Theorems 1.2 and 1.3. In Section 5 we outline a conjectural picture of formation of singularities of solutions to (1-1) in any dimension, and further clarify this picture in the case of complex surfaces. In Section 6 we show that (1-1) is a gradient flow, and show the existence of an entropy functional. In Section 7 we derive some consequences of the conjectural regularity picture developed in Section 5, and Section 8 is a brief conclusion.

\section{Acknowledgements}

The first named author would like to thank his advisor, Mark Stern, for first introducing him to the $B$-field renormalization group flow, and for many interesting conversations. He would also like to thank Nicholas Buchdahl for interesting conversations.

Streets was supported by DMS-1201569 and Tian was supported by DMS-0804095.

\section{$2 \quad L^{\infty}$ uniform metric estimate}

In this section we derive differential inequalities and produce a priori estimates for the metric along a solution to (1-1) that are similar in spirit to the Laplacian estimates for the solution to the complex Monge-Ampère equation. We will fix a one-parameter family of background metrics $\widetilde{\omega}(t)$ and assume that they are uniformly bounded on the time interval of consideration. Furthermore, we set

$$
\psi:=\frac{\partial}{\partial t} \tilde{\omega} .
$$

Finally, $\phi$ will always denote the solution to (1-3) taken with respect to $\widetilde{\omega}(t)$.

Lemma 2.1 Let $\left(M^{2 n}, \omega, J\right)$ be a complex manifold with pluriclosed metric. Then in local complex coordinates: $P^{1,1}(\omega)_{k \bar{l}}=g^{i \bar{j}}\left(-g_{k \bar{l}, i \bar{j}}+g^{m \bar{n}} g_{k \bar{n}, i} g_{\bar{l} m, \bar{j}}\right)-g^{m \bar{n}} g^{p \bar{q}}\left(g_{m \bar{q}, k}-g_{k \bar{q}, m}\right)\left(g_{\bar{n} p, \bar{l}}-g_{\bar{l} p, \bar{n}}\right)$

Proof As exhibited in [27], one has an expression

$$
P^{1,1}(\omega)_{k \bar{l}}=-S_{k \bar{l}}+Q_{k \bar{l}}^{1},
$$

where

$$
S_{k \bar{l}}=g^{i \bar{j}} \Omega_{i \bar{j} k \bar{l}} \quad \text { and } \quad Q_{k \bar{l}}^{1}=g^{m \bar{n}} g^{p \bar{q}} T_{k m \bar{q}} T_{\bar{l} \bar{n} p} \text {, }
$$


where $\Omega$ and $T$ are the curvature and torsion of the Chern connection, respectively. The lemma then follows from direct calculations.

Lemma 2.2 Let $\left(M^{2 n}, \widetilde{\omega}(t), J\right)$ be a complex manifold with a one-parameter family of pluriclosed metrics. Let $\omega(t)$ denote a solution to (1-1). Then in local complex coordinates:

$$
\begin{aligned}
& \frac{\partial}{\partial t} \operatorname{tr}_{\widetilde{\omega}} \omega=\tilde{g}^{k \bar{l}} g^{i \bar{j}}\left(g_{k \bar{l}, i \bar{j}}\right)-\widetilde{g}^{k \bar{l}} g^{i \bar{j}} g^{m \bar{n}} g_{k \bar{n}, i} g_{\bar{l} m, \bar{j}} \\
& +\tilde{g}^{k \bar{l}} g^{m \bar{n}} g^{p \bar{q}}\left(g_{m \bar{q}, k}-g_{k \bar{q}, m}\right)\left(g_{\bar{n} p, \bar{l}}-g_{\bar{l} p, \bar{n}}\right)-\langle\psi, \omega\rangle_{\widetilde{\omega}}, \\
& \frac{\partial}{\partial t} \log \frac{\omega^{n}}{\widetilde{\omega}^{n}}=\Delta \log \frac{\omega^{n}}{\widetilde{\omega}^{n}}+g^{k \bar{l}} g^{m \bar{n}} g^{p \bar{q}}\left(g_{m \bar{q}, k}-g_{k \bar{q}, m}\right)\left(g_{\bar{n} p, \bar{l}}-g_{\bar{l} p, \bar{n}}\right) \\
& +\operatorname{tr}_{\omega} \partial \bar{\partial} \log \operatorname{det} \tilde{g}-\operatorname{tr}_{\widetilde{\omega}} \psi, \\
& \frac{\partial}{\partial t} \operatorname{tr}_{\omega} \tilde{\omega}=g^{i \bar{q}} g^{p \bar{j}} g^{r \bar{s}}\left[-g_{p \bar{q}, r \bar{s}}+g^{u \bar{v}} g_{p \bar{v}, r} g_{u \bar{q}, \bar{s}}\right] \widetilde{g}_{i \bar{j}} \\
& -g^{i \bar{q}} g^{p \bar{j}} g^{r \bar{s}} g^{u \bar{v}}\left(g_{r \bar{v}, p}-g_{p \bar{v}, r}\right)\left(\partial_{\bar{q}} g_{u \bar{s}}-\partial_{\bar{s}} g_{u \bar{q}}\right) \tilde{g}_{i \bar{j}}+\operatorname{tr}_{\omega} \psi .
\end{aligned}
$$

Proof Starting from Lemma 2.1, we compute

$$
\begin{aligned}
\frac{\partial}{\partial t} \operatorname{tr}_{\widetilde{\omega}} \omega & =-\widetilde{g}^{k \bar{l}} P^{1,1}(\omega)_{k \bar{l}} \\
=\widetilde{g}^{k \bar{l}} g^{i \bar{j}}\left(g_{k \bar{l}, i \bar{j}}\right) & -\left\langle\psi, \omega \tilde{g}^{k \bar{l}} g^{i \bar{j}} g^{m \bar{n}} g_{k \bar{n}, i} g_{\bar{l} m, \bar{j}}\right. \\
& +\widetilde{g}^{k \bar{l}} g^{m \bar{n}} g^{p \bar{q}}\left(g_{m \bar{q}, k}-g_{k \bar{q}, m}\right)\left(g_{\bar{n} p, \bar{l}}-g_{\bar{l} p, \bar{n}}\right)-\langle\psi, \omega\rangle_{\widetilde{\omega}}
\end{aligned}
$$

Using a general calculation we have

$$
\frac{\partial}{\partial t} \log \frac{\omega^{n}}{\widetilde{\omega}^{n}}=\operatorname{tr}_{\omega}\left(\frac{\partial}{\partial t} \omega\right)-\operatorname{tr}_{\tilde{\omega}}\left(\frac{\partial}{\partial t} \widetilde{\omega}\right)
$$

Thus:

$$
\begin{aligned}
\frac{\partial}{\partial t} \log \frac{\omega^{n}}{\widetilde{\omega}^{n}}=g^{k \bar{l}} g^{i \bar{j}} g_{k \bar{l}, i \bar{j}}-g^{k \bar{l}} g^{i \bar{j}} g^{m \bar{n}} g_{k \bar{n}, i} g_{\bar{l} m, \bar{j}} \\
\quad+g^{k \bar{l}} g^{m \bar{n}} g^{p \bar{q}}\left(g_{m \bar{q}, k}-g_{k \bar{q}, m}\right)\left(g_{\bar{n} p, \bar{l}}-g_{\bar{l} p, \bar{n}}\right)-\operatorname{tr}_{\widetilde{\omega}} \psi \\
=\Delta \log \omega^{n}+g^{k \bar{l}} g^{m \bar{n}} g^{p \bar{q}}\left(g_{m \bar{q}, k}-g_{k \bar{q}, m}\right)\left(g_{\bar{n} p, \bar{l}}-g_{\bar{l} p, \bar{n}}\right)-\operatorname{tr}_{\widetilde{\omega}} \psi \\
=\Delta \log \frac{\omega^{n}}{\widetilde{\omega}^{n}}+g^{k \bar{l}} g^{m \bar{n}} g^{p \bar{q}}\left(g_{m \bar{q}, k}-g_{k \bar{q}, m}\right)\left(g_{\bar{n} p, \bar{l}}-g_{\bar{l} p, \bar{n}}\right) \\
+\operatorname{tr}_{\omega} \partial \bar{\partial} \log \operatorname{det} \tilde{g}-\operatorname{tr}_{\widetilde{\omega}} \psi
\end{aligned}
$$


Lastly we compute:

$$
\begin{aligned}
& \frac{\partial}{\partial t} \operatorname{tr}_{\omega} \widetilde{\omega}=g^{i \bar{q}} g^{p \bar{j}} P_{p \bar{q}}^{1,1} \widetilde{g}_{i \bar{j}}+\operatorname{tr}_{\omega} \psi \\
& =g^{i \bar{q}} g^{p \bar{j}} g^{r \bar{s}}\left[-g_{p \bar{q}, r \bar{s}}+g^{u \bar{v}} g_{p \bar{v}, r} g_{u \bar{q}, \bar{s}}\right] \widetilde{g}_{i \bar{j}} \\
& -g^{i \bar{q}} g^{p \bar{j}} g^{r \bar{s}} g^{u \bar{v}}\left(g_{r \bar{v}, p}-g_{p \bar{v}, r}\right)\left(\partial_{\bar{q}} g_{u \bar{s}}-\partial_{\bar{s}} g_{u \bar{q}}\right) \tilde{g}_{i \bar{j}}+\operatorname{tr}_{\omega} \psi
\end{aligned}
$$

We next record a lemma fixing certain canonical coordinates for $g$.

Lemma 2.3 [13, Lemma 2.1] Fix $\left(M^{2 n}, \tilde{g}, J\right)$ a complex manifold with Hermitian metric, and $g$ another Hermitian metric on $M$. For $p \in M$, there exist complex coordinates near $p$ such that

$$
\tilde{g}_{i \bar{j}}(p)=\delta_{i j}, \quad \partial_{j} \tilde{g}_{i \bar{i}}=0, \quad g_{i \bar{j}}=g_{i \bar{i}} \delta_{i j} .
$$

Proposition 2.4 Let $\left(M^{2 n}, \widetilde{\omega}(t), J\right)$ be a compact complex manifold with a oneparameter family of pluriclosed metrics, and suppose $\omega(t)$ is a solution of (1-1) and $\phi$ is a solution to (1-3). Fix a constant $A>0$ and let

$$
F=\log \operatorname{tr}_{\widetilde{\omega}} \omega-A \phi .
$$

There is a constant $C=C(\widetilde{g})$ such that

$$
\begin{aligned}
\left(\Delta-\frac{\partial}{\partial t}\right) F \geq-\sum_{i} \frac{1}{\operatorname{tr}_{\widetilde{\omega}} \omega} g^{m \bar{n}} g^{p \bar{q}}\left(g_{m \bar{q}, i}-g_{i \bar{q}, m}\right)\left(g_{\bar{n} p, \bar{i}}-g_{\bar{i} p, \bar{n}}\right) & \\
& +(A-C) \operatorname{tr}_{\omega} \widetilde{\omega}+\frac{\langle\psi, \omega\rangle_{\widetilde{\omega}}}{\operatorname{tr}_{\widetilde{\omega}} \omega}-A n .
\end{aligned}
$$

Proof Fix a point $p \in M$, choose coordinates for $\tilde{g}$ centered at $p$ as in Lemma 2.3 and compute

$$
\begin{aligned}
\Delta \operatorname{tr}_{\widetilde{\omega}} \omega=g^{i \bar{j}} \partial_{i} \partial_{\bar{j}}\left(\widetilde{g}^{k \bar{l}} g_{k \bar{l}}\right) & \\
& =\sum_{i, k} g^{i \bar{i}} g_{k \bar{k}, i \bar{i}}-2 \Re\left(\sum_{i, j, k} g^{i \bar{i}} \widetilde{g}_{j \bar{k}, \bar{i}} g_{k \bar{j}, i}\right)+\mathcal{O}\left(\left(\operatorname{tr}_{\omega} \widetilde{\omega}\right)\left(\operatorname{tr}_{\widetilde{\omega}} \omega\right)\right) .
\end{aligned}
$$

Plugging this calculation into the result of Lemma 2.2, we conclude:

$$
\begin{aligned}
&\left(\Delta-\frac{\partial}{\partial t}\right) \operatorname{tr} \widetilde{\omega} \omega=-2 \Re\left(\sum_{i, j, k} g^{i \bar{i}} \widetilde{g}_{j \bar{k}, i} g_{k \bar{j}, i}\right)+\sum_{k} g^{i \bar{i}} g^{m \bar{m}}\left(g_{k \bar{m}, i} g_{\bar{k} m, \bar{i}}\right) \\
&-g^{m \bar{n}} g^{p \bar{q}}\left(g_{m \bar{q}, i}-g_{i \bar{q}, m}\right)\left(g_{\bar{n} p, \bar{i}}-g_{\bar{i} p, \bar{n}}\right) \\
&+\mathcal{O}\left(\left(\operatorname{tr}_{\omega} \widetilde{\omega}\right)\left(\operatorname{tr}_{\widetilde{\omega}} \omega\right)\right)+\langle\psi, \omega\rangle_{\widetilde{\omega}} .
\end{aligned}
$$


Using the properties of Lemma 2.3 we can estimate:

$$
\left|-2 \Re \sum_{i, j, k} g^{i \bar{i}} \widetilde{g}_{j \bar{k}, \bar{i}} g_{k \bar{j}, i}\right| \leq \sum_{i, j \neq k} g^{i \bar{i}} g^{j \bar{j}} g_{k \bar{j}, i} g_{\bar{k} j, \bar{i}}+\mathcal{O}\left(\left(\operatorname{tr}_{\omega} \widetilde{\omega}\right)\left(\operatorname{tr}_{\widetilde{\omega}} \omega\right)\right)
$$

Next let us apply the properties of the coordinates in Lemma 2.3 and the CauchySchwarz inequality twice to yield:

$$
\begin{aligned}
\frac{\left|\partial \operatorname{tr}_{\widetilde{\omega}} \omega\right|_{g}^{2}}{\operatorname{tr}_{\tilde{\omega}} \omega} & =\frac{1}{\operatorname{tr}_{\widetilde{\omega}} \omega} \sum_{i, j, k} g^{i \bar{i}} \partial_{i} g_{j \bar{j}} \partial_{\bar{i}} g_{k \bar{k}} \\
& =\frac{1}{\operatorname{tr}_{\widetilde{\omega}} \omega} \sum_{j, k} \sum_{i} \sqrt{g^{i \bar{i}}} \partial_{i} g_{j \bar{j}} \sqrt{g^{i \bar{i}}} \partial_{\bar{i}} g_{k \bar{k}} \\
& \leq \frac{1}{\operatorname{tr}_{\widetilde{\omega}} \omega} \sum_{j, k}\left(\sum_{i} g^{i \bar{i}}\left|\partial_{i} g_{j \bar{j}}\right|^{2}\right)^{\frac{1}{2}}\left(\sum_{i} g^{i \bar{i}}\left|\partial_{i} g_{k \bar{k}}\right|^{2}\right)^{\frac{1}{2}} \\
& =\frac{1}{\operatorname{tr}_{\widetilde{\omega}} \omega}\left(\sum_{j}\left(\sum_{i} g^{i \bar{i}}\left|\partial_{i} g_{j}\right|_{j}^{2}\right)^{\frac{1}{2}}\right)^{2} \\
& =\frac{1}{\operatorname{tr}_{\widetilde{\omega}} \omega}\left(\sum_{j} \sqrt{g_{j} \bar{j}}\left(\sum_{i} g^{i \bar{i}} g^{j \bar{j}}\left|\partial_{i} g_{j \bar{j}}\right|^{2}\right)^{\frac{1}{2}}\right)^{2} \leq \sum_{i, j} g^{i i \bar{i}} g^{j \bar{j}} \partial_{i} g_{j \bar{j}} \partial_{\bar{i}} g_{j} \bar{j}
\end{aligned}
$$

Finally we can now conclude:

$$
\begin{aligned}
& \sum_{k} g^{i \bar{i}} g^{m \bar{m}}\left(g_{k \bar{m}, i} g_{\bar{k} m, \bar{i}}\right)-\frac{|\partial \operatorname{tr} \widetilde{\omega} g|_{g}^{2}}{\operatorname{tr}_{\widetilde{\omega}} \omega}-2 \Re\left(\sum_{i, j, k} g^{i \bar{i}} \tilde{g}_{j \bar{k}, \bar{i}} g_{k \bar{j}, i}\right) \\
& \geq-\mathcal{O}\left(\left(\operatorname{tr}_{\omega} \widetilde{\omega}\right)\left(\operatorname{tr}_{\widetilde{\omega}} \omega\right)\right)
\end{aligned}
$$

Combining the above calculations yields the result.

Proposition 2.5 Let $\left(M^{2 n}, \widetilde{\omega}(t), J\right)$ be a compact complex manifold with a oneparameter family of pluriclosed metrics and suppose $\omega(t)$ is a solution of (1-1), and $\phi$ is a solution to (1-3). Fix a constant $A>0$ and let

$$
F=\log \operatorname{tr}_{\widetilde{\omega}} \omega-\log \frac{\omega^{n}}{\widetilde{\omega}^{n}}-A \phi .
$$

There is a constant $C=C(\widetilde{g})$ such that

$$
\left(\Delta-\frac{\partial}{\partial t}\right) F \geq(A-C) \operatorname{tr}_{\omega} \widetilde{\omega}-\frac{\langle\psi, \omega\rangle_{\tilde{\omega}}}{\operatorname{tr}_{\widetilde{\omega}} \omega}-\operatorname{tr}_{\widetilde{\omega}} \psi-A n
$$


Proof Starting from the result of Proposition 2.4 and using Lemma 2.2 we immediately conclude:

$$
\begin{aligned}
&\left(\Delta-\frac{\partial}{\partial t}\right) F \geq g^{k \bar{l}} g^{m \bar{n}} g^{p \bar{q}}\left(g_{m \bar{q}, k}-g_{k \bar{q}, m}\right)\left(g_{\bar{n} p, \bar{l}}-g_{\bar{l} p, \bar{n}}\right) \\
&-\frac{1}{\operatorname{tr}_{\tilde{\omega}} \omega} g^{m \bar{n}} g^{p \bar{q}}\left(g_{m \bar{q}, i}-g_{i \bar{q}, m}\right)\left(g_{\bar{n} p, \bar{i}}-g_{\bar{i} p, \bar{n}}\right) \\
&+(A-C) \operatorname{tr}_{\omega} \tilde{\omega}+\frac{\langle\psi, \omega\rangle_{\widetilde{\omega}}}{\operatorname{tr}_{\widetilde{\omega}} \omega}-\operatorname{tr}_{\widetilde{\omega}} \psi-A n
\end{aligned}
$$

Let $T_{k m \bar{q}}=g_{m \bar{q}, k}-g_{k \bar{q}, m}$. The first term above is then $|T|^{2}$. Likewise, if we choose coordinates at a point such that $\widetilde{g}_{i \bar{j}}=\delta_{i j}$ and $g$ is diagonalized, specifically $g_{i \bar{i}}=\lambda_{i}$, then

$$
\frac{1}{\operatorname{tr}_{\widetilde{\omega}} \omega} \leq \frac{1}{\lambda_{i}}
$$

for any $i$. Thus:

$$
\begin{aligned}
-\frac{1}{\operatorname{tr}_{\tilde{\omega}} \omega} g^{m \bar{n}} g^{p \bar{q}}\left(g_{m \bar{q}, i}-g_{i \bar{q}, m}\right)\left(g_{\bar{n} p, \bar{i}}-g_{\bar{i} p, \bar{n}}\right) & =-\frac{1}{\operatorname{tr}_{\widetilde{\omega}} \omega} \sum_{i} g^{m \bar{n}} g^{p \bar{q}} T_{i m \bar{q}} T_{\bar{i} \bar{n} p} \\
& \geq-\sum_{i} g^{i \bar{i}} g^{m \bar{n}} g^{p \bar{q}} T_{i m \bar{q}} T_{\bar{i} \bar{n} p} \\
& =-|T|^{2}
\end{aligned}
$$

The proposition follows.

Proposition 2.6 Let $\left(M^{2 n}, \widetilde{\omega}(t), J\right)$ be a compact complex manifold with a oneparameter family of pluriclosed metrics and suppose $\omega(t)$ is a solution of (1-1). Then in local complex coordinates:

$$
\begin{aligned}
&\left(\Delta-\frac{\partial}{\partial t}\right) \operatorname{tr}_{\omega} \tilde{\omega}=g^{r \bar{s}} g^{i \bar{v}} g^{u \bar{q}} g^{p \bar{j}} g_{u \bar{v}, r} g_{p \bar{q}, \bar{s}} \widetilde{g}_{i \bar{j}} \\
&-2 \Re\left(g^{r \bar{s}} g^{i \bar{q}} g^{p \bar{j}} g_{p \bar{q}, \bar{s}} \widetilde{g}_{i \bar{j}, r}\right)+g^{r \bar{s}} g^{i \bar{j}} \widetilde{g}_{i \bar{j}, r \bar{s}} \\
& \quad+g^{i \bar{q}} g^{p \bar{j}} g^{r \bar{s}} g^{u \bar{v}}\left(g_{r \bar{v}, p}-g_{p \bar{v}, r}\right)\left(g_{u \bar{s}, \bar{q}}-g_{u \bar{q}, \bar{s}}\right) \widetilde{g}_{i \bar{j}}-\operatorname{tr}_{\omega} \psi
\end{aligned}
$$

Proof We directly compute:

$$
\begin{aligned}
\Delta \operatorname{tr}_{\omega} \widetilde{\omega}= & g^{r \bar{s}} \partial_{r} \partial_{\bar{s}}\left[g^{i} \bar{j} \widetilde{g}_{i \bar{j}}\right] \\
= & g^{r \bar{s}} \partial_{r}\left[-g^{i \bar{q}} g^{p \bar{j}} g_{p \bar{q}, \bar{s}} \widetilde{g}_{i \bar{j}}+g^{i \bar{j}} \widetilde{g}_{i \bar{j}, \bar{s}}\right] \\
= & g^{r \bar{s}}\left[g^{i \bar{v}} g^{u \bar{q}} g^{p \bar{j}} g_{u \bar{v}, r} g_{p \bar{q}, \bar{s}} \widetilde{g}_{i \bar{j}}+g^{i \bar{q}} g^{p \bar{v}} g^{u \bar{j}} g_{u \bar{v}, r} g_{p \bar{q}, \bar{s}} \widetilde{g}_{i \bar{j}}\right. \\
& \left.-g^{i \bar{q}} g^{p \bar{j}} g_{p \bar{q}, r \bar{s}} \widetilde{g}_{i \bar{j}}-g^{i \bar{q}} g^{p \bar{j}} g_{p \bar{q}, \bar{s}} \widetilde{g}_{i \bar{j}, r}-g^{i \bar{q}} g^{p \bar{j}} g_{p \bar{q}, r} \widetilde{g}_{i \bar{j}, \bar{s}}+g^{i \bar{j}} \widetilde{g}_{i \bar{j}, r \bar{s}}\right]
\end{aligned}
$$


Combining this with the result of Lemma 2.2 yields the result.

Proposition 2.7 Let $\left(M^{2 n}, \widetilde{\omega}(t), J\right)$ be a compact complex manifold with a oneparameter family of pluriclosed metrics and suppose $\omega(t)$ is a solution of (1-1) on $[0, \tau)$, and $\phi$ is a solution to (1-3). There is a constant $C=C(\widetilde{g}, \psi)>0$ such that

$$
\operatorname{tr}_{\widetilde{\omega}} \omega \leq C e^{C\left(t+\log \left(\omega^{n} / \widetilde{\omega}^{n}\right)+\phi\right)} .
$$

Proof Let $F=\log \operatorname{tr}_{\widetilde{\omega}} \omega-\log \left(\omega^{n} / \widetilde{\omega}^{n}\right)-A \phi-B t$, where the constants $A$ and $B$ are to be determined. Using Proposition 2.5 we obtain:

$$
\left(\Delta-\frac{\partial}{\partial t}\right) F \geq(A-C) \operatorname{tr}_{\omega} \widetilde{\omega}-\frac{\langle\psi, \omega\rangle_{\widetilde{\omega}}}{\operatorname{tr}_{\widetilde{\omega}} \omega}-\operatorname{tr}_{\widetilde{\omega}} \psi-A n+B
$$

Now we note that $\left|\langle\psi, \omega\rangle_{\widetilde{\omega}}\right| \leq|\psi|_{\widetilde{\omega}}|\omega|_{\widetilde{\omega}} \leq C|\psi|_{\widetilde{\omega}} \operatorname{tr}_{\widetilde{\omega}} \omega$. Thus we have:

$$
\left(\Delta-\frac{\partial}{\partial t}\right) F \geq(A-C-C|\psi| \tilde{\omega})-A n+B
$$

Choosing $A$ sufficiently large with respect to the constants and $|\psi|_{\widetilde{\omega}}$ and then choosing $B=n A$ yields

$$
\left(\Delta-\frac{\partial}{\partial t}\right) F \geq 0
$$

By the maximum principle, for any $t<\tau$ we conclude $\sup _{M} F(t) \leq \sup _{M} F(0)$. The result follows.

\section{$3 C^{1}$ metric estimate}

In this section we derive certain differential inequalities for the Chern connection along a solution to (1-1). These estimates are inspired by Calabi's third-order estimate of the potential function for the complex Monge-Ampère equation, and similar estimates for the Kähler Ricci flow were considered in Phong, Sesum and Sturm [23]. Fix $\left(M^{2 n}, \tilde{g}, J\right)$ a Hermitian manifold and let $g$ denote another Hermitian metric on $M$. Let $h$ denote the endomorphism of the tangent bundle:

$$
h_{j}^{i}=\widetilde{g}^{i \bar{k}} g_{\bar{k} j}
$$

Let

$$
\Upsilon=\nabla h h^{-1} \quad \text { and } \quad W=|\Upsilon|^{2}=g^{i \bar{j}} g^{k \bar{l}} g_{m \bar{n}} \Upsilon_{i k}^{m} \overline{\Upsilon_{j l}^{n}},
$$

where the first lowered index on the tensor $\Upsilon=\nabla h h^{-1}$ is that arising from the derivative. The tensor $\Upsilon$ is the difference of the Chern connections induced by $\tilde{g}$ and $g$. In particular one observes that

$$
\bar{\nabla} \Upsilon=\widetilde{\Omega}-\Omega .
$$


Lemma 3.1 Let $\left(M^{2 n}, \widetilde{g}, J\right)$ be a Hermitian manifold, let $g$ denote another Hermitian metric on $M$, and let $W$ be defined as above. Then:

$$
\begin{aligned}
\Delta W=|\bar{\nabla} \Upsilon|^{2} & +|\nabla \Upsilon|^{2} \\
& +g^{i \bar{j}} g^{k \bar{l}} g_{m \bar{n}} \overline{\left(-g^{\bar{q} p} T_{j p}^{r} \Omega_{\bar{q} r l}^{n}-\nabla_{j} S_{l}^{n}+g{ }^{p \bar{q}} \nabla_{p} \widetilde{\Omega}_{\bar{q} j l}^{n}\right)} \Upsilon_{i k}^{m}+\text { conjugate } \\
& +\Upsilon_{i k}^{m}\left(S^{i \bar{r}} g^{k \bar{l}} g_{m \bar{n}} \overline{\Upsilon_{r l}^{n}}+g^{i \bar{j}} S^{k \bar{r}} g_{m \bar{n}} \overline{\Upsilon_{j r}^{n}}-g^{i} g^{k \bar{l}} S_{r \bar{m}} \overline{\Upsilon_{j l}^{m}}\right)
\end{aligned}
$$

Proof First we compute

$$
\Delta W=g^{i \bar{j}} g^{k \bar{l}} g_{m \bar{n}}\left[\Delta \Upsilon_{i k}^{m} \overline{\Upsilon_{j l}^{n}}+\Upsilon_{i k}^{m} \overline{\bar{\Delta} \Upsilon_{j l}^{n}}\right]+|\bar{\nabla} \Upsilon|^{2}+|\nabla \Upsilon|^{2} .
$$

Next we commute derivatives to see:

$$
\begin{aligned}
\bar{\Delta} \Upsilon_{j l}^{n} & =g^{p \bar{q}} \nabla_{\bar{q}} \nabla_{p} \Upsilon_{j l}^{n} \\
& =g^{p \bar{q}}\left[\nabla_{p} \nabla_{\bar{q}} \Upsilon_{j l}^{n}-\Omega_{\bar{q} p j}^{r} \Upsilon_{r l}^{n}-\Omega_{\bar{q} p l}^{r} \Upsilon_{j r}^{n}+\Omega_{\bar{q} p r}^{n} \Upsilon_{j l}^{r}\right] \\
& =\Delta \Upsilon_{j l}^{n}+S_{j}^{r} \Upsilon_{r l}^{n}+S_{l}^{r} \Upsilon_{j r}^{n}-S_{r}^{n} \Upsilon_{j l}^{r}
\end{aligned}
$$

Finally we observe using a general formula for curvatures of Hermitian metrics that:

$$
\begin{aligned}
\Delta \Upsilon_{j l}^{n} & =g^{\bar{q} p} \nabla_{p} \nabla_{\bar{q}} \Upsilon_{j l}^{n} \\
& =g^{\bar{q} p} \nabla_{p}\left(\widetilde{\Omega}_{\bar{q} j l}^{n}-\Omega_{\bar{q} j l}^{n}\right) \\
& =g^{\bar{q} p}\left(-\nabla_{j} \Omega_{\bar{q} p l}^{n}-T_{j p}^{r} \Omega_{\bar{q} r l}^{n}+\nabla_{p} \widetilde{\Omega}_{\bar{q} j l}^{n}\right) \\
& =-\nabla_{j} S_{l}^{n}-g^{\bar{q} p} T_{j p}^{r} \Omega_{\bar{q} r l}^{n}+g^{\bar{q} p} \nabla_{p} \tilde{\Omega}_{\bar{q} j l}^{n}
\end{aligned}
$$

Combining these calculations yields the result.

Proposition 3.2 Let $\left(M^{2 n}, \widetilde{g}, J\right)$ be a Hermitian manifold, let $g$ denote a solution to (1-1), and let $W$ be defined as above. Then:

$$
\begin{aligned}
& \left(\Delta-\frac{\partial}{\partial t}\right) W=|\bar{\nabla} \Upsilon|^{2}+|\nabla \Upsilon|^{2} \\
& +g^{i \bar{j}} g^{k \bar{l}} g_{m \bar{n}} \overline{\left(-g^{\bar{q} p} T_{j p}^{r} \Omega_{\bar{q} r l}^{n}-\nabla_{j} Q_{l}^{n}+g^{p \bar{q}} \nabla_{p} \widetilde{\Omega}_{\bar{q} j l}^{n}\right)} \Upsilon_{i k}^{m} \\
& + \text { conjugate } \\
& +\Upsilon_{i k}^{m}\left(\left(Q^{1}\right)^{i \bar{r}} g^{k \bar{l}} g_{m \bar{n}} \overline{\Upsilon_{r l}^{n}}+g^{i \bar{j}}\left(Q^{1}\right)^{k \bar{r}} g_{m \bar{n}} \overline{\Upsilon_{j r}^{n}}-g^{i \bar{j}} g^{k \bar{l}} Q_{r \bar{m}}^{1} \overline{\Upsilon_{j l}^{m}}\right)
\end{aligned}
$$

Proof First observe the variational equation:

$$
\frac{\partial}{\partial t}\left(\nabla h h^{-1}\right)=\nabla\left(h^{-1}\left(\frac{\partial}{\partial t} h\right)\right)
$$


It follows from Lemma 3.1 that for a general variation one has:

$$
\begin{aligned}
& \left(\Delta-\frac{\partial}{\partial t}\right) W \\
& =|\bar{\nabla} \Upsilon|^{2}+|\nabla \Upsilon|^{2} \\
& \quad+g^{i \bar{j}} g^{k \bar{l}} g_{m \bar{n}} \overline{\left(-g^{\bar{q} p} T_{j p}^{r} \Omega_{\bar{q} r l}^{n}-\nabla_{j} S_{l}^{n}-\nabla_{j}\left(h^{-1} \dot{h}\right)_{l}^{n}+g^{p \bar{q}} \nabla_{p} \widetilde{\Omega}_{\bar{q} j l}^{n}\right)} \Upsilon_{i k}^{m} \\
& + \text { conjugate } \\
& +\Upsilon_{i k}^{m}\left(\left(h^{-1} \dot{h}^{i \bar{r}}+S^{i \bar{r}}\right) g^{k \bar{l}} g_{m \bar{n}} \overline{\Upsilon_{r l}^{n}}\right. \\
& \left.\quad+g^{i \bar{j}}\left(\left(h^{-1} \dot{h}\right)^{k \bar{r}}+S^{k \bar{r}}\right) g_{m \bar{n}} \overline{\Upsilon_{j r}^{n}}-g^{i \bar{j}} g^{k \bar{l}}\left(h^{-1} \dot{h}_{r \bar{m}}+S_{r \bar{m}}\right) \overline{\Upsilon_{j l}^{m}}\right)
\end{aligned}
$$

Plugging in $\dot{g}=-P^{1,1}=-S+Q^{1}$ yields the result.

Proposition 3.3 Let $\left(M^{2 n}, \tilde{g}, J\right)$ be a Hermitian manifold, let $g$ denote a solution to $(1-1)$ on $[0, \tau)$, and let $W$ be defined as above. Suppose there exists a constant $K$ such that

$$
\frac{1}{K} \tilde{g} \leq g(t) \leq K \widetilde{g}
$$

for all $t \in[0, \tau)$. Then there is a constant $C(K, \widetilde{g})$ such that:

$$
\left(\Delta-\frac{\partial}{\partial t}\right) W \geq-C\left(1+W+|T|^{2} W\right)
$$

Proof We start by noting that $\bar{\nabla} \Upsilon=\Omega-\widetilde{\Omega}$; thus, $|\bar{\nabla} \Upsilon|^{2} \geq \frac{1}{2}|\Omega|^{2}-C|\widetilde{\Omega}|^{2}$. Also, by orthogonally projecting $\Upsilon$ onto its skew symmetric part, one observes that:

$$
\begin{aligned}
|\bar{\nabla} \Upsilon|^{2}+|\nabla \Upsilon|^{2} & \geq \frac{1}{2}|\bar{\nabla} T|^{2}+\frac{1}{2}|\nabla T|^{2}-C|\nabla \tilde{T}|^{2} \\
& \geq \frac{1}{2}|\bar{\nabla} T|^{2}+\frac{1}{2}|\nabla T|^{2}-C W
\end{aligned}
$$

Thus, starting from the result of Proposition 3.2 we first estimate using the CauchySchwarz inequality:

$$
\begin{aligned}
g^{i \bar{j}} g^{k \bar{l}} g_{m \bar{n}} g^{p \bar{q}} \nabla_{p} \tilde{\Omega}_{\bar{q} j l}^{n} \Upsilon_{i k}^{m} & =g * g^{-1}(\tilde{\nabla} \tilde{\Omega}+\Upsilon * \widetilde{\Omega}) * \Upsilon \\
& \leq C(K)\left[|\tilde{\nabla} \tilde{\Omega}|_{g}^{2}+W\right] \leq C(K, \tilde{g})(1+W)
\end{aligned}
$$

Next we estimate:

$$
\begin{aligned}
\left|g^{i \bar{j}} g^{k \bar{l}} g_{m \bar{n}} g^{p \bar{q}} T_{j p}^{r} \Omega_{\bar{q} r l}^{n} \Upsilon_{i k}^{m}\right| & \leq C|T||\Omega||\Upsilon| \\
& \leq \theta|\Omega|^{2}+\frac{C}{\theta}|T|^{2}|\Upsilon|^{2} \\
& \leq \theta|\Omega|^{2}+\frac{C}{\theta}|T|^{2} W
\end{aligned}
$$


Similarly, we have:

$$
\begin{aligned}
\left|g^{i \bar{j}} g^{k \bar{l}} g_{m \bar{n}} \nabla_{j} Q_{l}^{n} \Upsilon_{i k}^{n}\right| & \leq C[|\nabla T|+|\bar{\nabla} T|]|T||\Upsilon| \\
& \leq \theta\left[|\bar{\nabla} T|^{2}+|\nabla T|^{2}\right]+\frac{C}{\theta}|T|^{2} W
\end{aligned}
$$

Finally, one clearly has

$$
\Upsilon_{i k}^{m} Q^{i \bar{r}} g^{k \bar{l}} g_{m \bar{n}} \overline{\Upsilon_{r l}^{n}} \leq C|T|^{2} W,
$$

and likewise for the rest of the terms. Choosing $\theta$ sufficiently small and combining these estimates yields the result.

\section{Regularity theorems}

In this section we give the proofs of the regularity theorems stated in the introduction. We start by giving the proof of Theorem 1.2.

Lemma 4.1 Let $\left(M^{2 n}, \omega(t), J\right)$ be a solution to (1-1) on a finite time interval $[0, \tau)$ and suppose:

$$
\int_{0}^{\tau}\left|\frac{\partial g}{\partial t}\right|<\infty
$$

Then there is a $C^{0}$ metric $\omega(\tau)$ such that $\lim _{t \rightarrow \tau} \omega(t)=\omega(\tau)$ in $C^{0}$.

Proof This is in Hamilton [14, Lemma 14.2].

Proposition 4.2 Let $\left(M^{2 n}, \omega(t), J\right)$ be a solution to (1-1) on $[0, \tau)$. Suppose $\omega(t) \rightarrow$ $\omega(\tau)$ in $C^{0}$, ie, the limit at time $\tau$ exists as a $C^{0}$ metric. Then in fact $\omega(t)$ is bounded in $C^{1}$ and for all $p<\infty$ there are constants $C_{p}$ such that

$$
\int_{M}\left|\Omega\left(\omega_{\tau}\right)\right|^{p} \leq C_{p}
$$

Proof For the $C^{1}$ norm on metrics we choose finitely many local coordinate patches to cover $M$ and take the supremum over the coordinate derivatives in all these charts. It is equivalent to choose a fixed connection $\nabla_{0}$ and use the covariant derivative with respect to that fixed connection.

Suppose that $\{\omega(t)\}$ is unbounded in $C^{1}$. Let $\phi(x, t)=\left|\nabla_{0} \omega\right|$. Then for some sequence $\left(x_{i}, t_{i}\right)$, where $t_{i} \rightarrow \tau$, we have $\sup _{M} \phi\left(t_{i}\right)$ is achieved at $x_{i}$, and moreover goes to infinity. By choosing a subsequence we may assume that $x_{i}$ converges to some 
point $x \in M$. By choosing a coordinate chart around $x$, and translating coordinates for $i$ sufficiently large we may assume that $\sup _{z} \phi=\alpha_{i}$ is attained at $z=0$. Now choose new coordinates $w=\alpha_{i} z$. Since $\omega$ is converging in $C^{0}$, it follows that

$$
\lim _{i \rightarrow \infty} \int_{\{|w|<1\}} \phi=0 \text {. }
$$

One may express the system (1-1) in local coordinates as:

$$
\frac{\partial}{\partial t} \omega_{i j}-g^{k l} \partial_{k} \partial_{l} \omega_{i j}=\omega^{-1} * \omega^{-1}\left(\partial \omega^{* 2}\right)
$$

The right hand side of the equation is uniformly bounded in $C^{0}$ on $[0, \tau)$, hence each coordinate function is the solution to a uniformly parabolic equation with continuous coefficients and bounded right hand side. It follows by Lieberman [19, Theorem 7.13] that on $\{|w|<1-\epsilon\}$ we have $|\omega|_{H_{2}^{p}}<\infty$ for all $p$.

Choosing $p>2 n$, and applying the Sobolev inequality, we attain a uniform $C^{1}$ bound for $\omega\left(t_{i}\right)$, and moreover a convergent subsequence at time $\tau$. But then, for this subsequence, (4-1) implies that $\lim _{i \rightarrow \infty} \phi\left(t_{i}\right)_{w=0}=0$, a contradiction. It follows that $\omega(t)$ is bounded in $C^{1}$. Now applying the above regularity argument to $\omega$ in local coordinates around any point yields again the $H_{2}^{p}$ bound on $\omega$ for all $p$, and hence the curvature $\Omega$ is bounded in $L^{p}$ for all $p$.

Proof of Theorem 1.2 We now proceed with the main argument. Suppose that the statement of the theorem were false. Then let $\left(M^{2 n}, \omega(t), J\right)$ be a solution to (1-1) on $[0, \tau)$ satisfying

$$
\int_{0}^{\tau}\left|\frac{\partial g}{\partial t}\right|<\infty
$$

By Lemma 4.1 we conclude the existence of a $C^{0}$ limit metric $\omega(\tau)$. By Proposition 4.2 we conclude that in fact $\omega(t)$ is a $C^{1}$ metric and furthermore one has uniform $L^{p}$ bounds on the curvature as $t \rightarrow \tau$. By [28, Theorem 1.1], if we can show a uniform bound on the $C^{0}$ norm of curvature and torsion, we can conclude smooth convergence of the metrics as $t \rightarrow \tau$. By the general short-time existence result for these equations, we conclude that $\tau$ is not the maximal existence time, providing the contradiction.

So, we can differentiate (4-2) to yield:

$$
\frac{\partial}{\partial t}\left(\nabla_{0} \omega\right)_{i j k}-g^{p q} \partial_{p} \partial_{q}\left(\nabla_{0} \omega\right)_{i j k}=\omega^{-1} * \omega^{-1} *\left(\partial \omega * \partial^{2} \omega\right)
$$

By the discussion above, the right hand side is uniformly bounded in $L^{p}$, so again we conclude that $\omega$ is uniformly bounded in $H_{3}^{p}$. Choosing $p$ sufficiently large and 
applying the Sobolev inequality, we conclude a uniform $C^{0}$ bound on $\Omega, T$ and $\nabla T$, and the theorem follows.

Now we give the proof of Theorem 1.3.

Proof Consider the differential inequality of Proposition 2.4. First we note that

$$
\frac{1}{\operatorname{tr}_{\widetilde{\omega}} \omega} g^{m \bar{n}} g^{p \bar{q}}\left(g_{m \bar{q}, i}-g_{i \bar{q}, m}\right)\left(g_{\bar{n} p, \bar{i}}-g_{\bar{i} p, \bar{n}}\right) \leq|T|^{2} .
$$

Applying the maximum principle, since $\phi$ and $|T|^{2}$ are bounded we conclude there is a uniform upper bound on $\operatorname{tr}_{\widetilde{\omega}} \omega$. In particular, there is a constant $K$ such that

$$
g(t) \leq K \tilde{g} .
$$

Also, by using Lemma 2.2 , if $F=\log (\operatorname{det} g / \operatorname{det} \tilde{g})+A \phi$, we compute that

$$
\frac{\partial}{\partial t} F \geq \Delta F+|T|^{2}+(A-C) \operatorname{tr}_{\omega} \tilde{\omega}-n .
$$

In particular, for $A$ chosen sufficiently large we conclude by the maximum principle

$$
\inf _{M \times[0, \tau)} \log \frac{\operatorname{det} g}{\operatorname{det} \widetilde{g}} \geq-C(|\phi|) .
$$

We thus conclude there is a uniform lower bound for $g(t)$ on $[0, \tau)$ by the arithmeticgeometric mean. Again using that the torsion is bounded, we may apply the maximum principle to the differential inequality in Proposition 3.3 to conclude there is a uniform $C^{1}$ bound on $g(t)$ on $[0, \tau)$. Equation $(1-1)$ is strictly parabolic in local complex coordinates, with bounded $C^{1}$ norm, so uniform $C^{k}$ estimates follow from the Schauder theory for all $k$, and the theorem follows.

Let us finish this section with a few remarks on the nature of the potential function $\phi$. First, Theorem 1.3 even provides a slightly different perspective on the regularity of Kähler Ricci flow. In this case the torsion vanishes for all time, so one only has to check that the potential function is bounded. It is clear by applying the maximum principle to (1-3) that if

$$
\int_{0}^{\tau} \sup _{M} \operatorname{tr}_{\omega} \tilde{\omega}<\infty
$$

then the flow will extend past time $\tau$. This condition can be checked in certain settings. Also, in the general non-Kähler setting the function $\phi$ is automatically bounded on certain background manifolds. Consider the following lemma. 
Lemma 4.3 Let $\left(M^{2 n}, \widetilde{\omega}, J\right)$ be a complex manifold and suppose $\widetilde{\omega}$ is Kähler and moreover $\widetilde{\Omega} \leq 0$, in the sense of sections of $\operatorname{End}\left(\Lambda^{1,1}\right)$. Let $\omega(t)$ denote a solution to (1-1) on $[0, \tau)$. Then there is a constant $C>0$ so that

$$
\begin{aligned}
\operatorname{tr}_{\omega} \tilde{\omega} & \leq C, \\
|\phi| & \leq C \tau .
\end{aligned}
$$

Proof From Proposition 2.6, if $\widetilde{\omega}$ is Kähler we can choose coordinates where $\partial_{i} \widetilde{g}_{j \bar{k}}=$ 0 and simplify, since $Q^{1} \geq 0$ :

$$
\begin{aligned}
\left(\Delta-\frac{\partial}{\partial t}\right) \operatorname{tr}_{\omega} \tilde{\omega} & \geq-g^{r \bar{s}} g^{i \bar{j}} \widetilde{\Omega}_{i \bar{j} r \bar{s}} \\
& \geq 0 .
\end{aligned}
$$

Applying the maximum principle proves the uniform upper bound for $\operatorname{tr}_{\omega} \widetilde{\omega}$, and then the bound for $\phi$ follows immediately applying the maximum principle to (1-3).

Since $\widetilde{\omega}$ is Kähler, $P$ is just the Ricci form, hence the hypotheses are satisfied on complex tori or Kähler manifolds with negative curvature operator. Note of course that we are not assuming $\omega$ is Kähler. This bound suggests that the function $\phi$ is quite natural to introduce, and furthermore suggests that its possible blowup is not related to any "local" singularity model since it is bounded on these natural background manifolds.

\section{Conjectural picture of singularity formation}

The notion of the Kähler cone in $H^{2}(M, \mathbb{R}) \cap H^{1,1}(M, \mathbb{C})$ is crucial to understanding the structure of solutions of Kähler Ricci flow. Recall from the introduction that along a solution to Kähler Ricci flow the Kähler class satisfies an ODE, depending on the normalization. Clearly a necessary condition for existence of the flow is that this ODE stay in the Kähler cone. As mentioned in the introduction, this condition is in fact sufficient [32, Proposition 1.1]. It is natural to conjecture that a similar phenomenon is at play guiding the singular behavior of solutions of (1-1).

First of all recall from the introduction that:

$$
\mathcal{H}_{\partial+\bar{\partial}}^{1,1}=\frac{\left\{\operatorname{Ker} \partial \bar{\partial}: \Lambda_{\mathbb{R}}^{1,1} \rightarrow \Lambda_{\mathbb{R}}^{2,2}\right\}}{\left\{\partial \alpha+\bar{\partial} \bar{\alpha} \mid \alpha \in \Lambda^{0,1}\right\}}
$$

This is known as the $(1,1)$ Aeppli cohomology group and one basic fact is that this space is finite-dimensional, as can be seen by constructing the necessary short exact 
sequence of coherent sheaves. Let the positive cone inside $\mathcal{H}_{\partial+\bar{\partial}}^{1,1}$ be:

$$
\mathcal{P}_{\partial+\bar{\partial}}=\left\{[\phi] \in \mathcal{H}_{\partial+\bar{\partial}}^{1,1} \mid \exists \psi \in[\phi], \psi>0\right\}
$$

It is clear that a necessary condition for a solution to (1-1) to exist is that the class $\left[\omega_{t}\right]=\left[\omega_{0}-t c_{1}\right] \in \mathcal{P}_{\partial+\bar{\partial}}$. We state this for emphasis.

Proposition 5.1 Let $\left(M^{2 n}, g_{0}, J\right)$ be a compact complex manifold with pluriclosed metric. Let:

$$
\tau^{*}:=\sup _{t \geq 0}\left\{t \mid\left[\omega_{0}-t c_{1}\right] \in \mathcal{P}_{\partial+\bar{\partial}}\right\}
$$

Let $\tau$ denote the maximal existence time of the solution of (1-1) with initial condition $g_{0}$. Then

$$
\tau \leq \tau^{*}
$$

Furthermore, in analogy with Kähler Ricci flow, it is natural to conjecture that membership in this cone suffices for existence.

Conjecture 5.2 (Weak existence conjecture) Let $\left(M^{2 n}, g_{0}, J\right)$ be a compact complex manifold with pluriclosed metric. Let:

$$
\tau^{*}:=\sup _{t \geq 0}\left\{t \mid\left[\omega_{0}-t c_{1}\right] \in \mathcal{P}_{\partial+\bar{\partial}}\right\}
$$

Then the solution to (1-1) with initial condition $g_{0}$ exists on $\left[0, \tau^{*}\right)$, and $\tau^{*}$ is the maximal time of existence.

Let us note here that this we are implicitly making this conjecture, and the two related conjectures below, for any normalization of (1-1), ie, the volume normalized version of (1-1) or other possible normalizations. A stronger version of this conjecture would be that there are uniform $C^{\infty}$ estimates on $\omega(t)$ depending on $d\left([\omega(t)], \partial \mathcal{P}_{\partial+\bar{\partial}}\right)$, where this means distance with respect to some metric defined on $\mathcal{H}_{\partial+\bar{\partial}}^{1,1}$. Let us also state this for emphasis.

Conjecture 5.3 (Strong existence conjecture) Let $\left(M^{2 n}, g_{0}, J\right)$ be a compact complex manifold with pluriclosed metric. Let $\omega(t)$ be the solution to (1-1) with initial condition $\omega_{0}$. Let $t$ be such that $\left[\omega_{0}-t c_{1}\right] \in \mathcal{P}_{\partial+\bar{\partial}}$. Then there exist uniform $C^{\infty}$ estimates on $\omega(t)$ depending on $\omega_{0}, t$ and $d\left([\omega(t)], \partial \mathcal{P}_{\partial+\bar{\partial}}\right)$. Moreover, there exist uniform bounds on the curvature and diameter of $\omega(t)$. 
It is possible to characterize $\mathcal{P}_{\partial+\bar{\partial}}$ using more calculable cohomological criteria in the case of non-Kähler complex surfaces, which will allow us to derive some consequences of Conjecture 5.3. Our Theorem 5.6 follows by combining the positivity result of Buchdahl [4] on non-Kähler surfaces (see also Lamari [18]), and further related results of Teleman [30]. Let us start by stating the main theorem of Buchdahl [5], which represents the main technical difficulty of Theorem 5.6.

Theorem 5.4 [5, Main Theorem] Let $\left(M^{4}, \omega, J\right)$ be a complex surface with pluriclosed metric $\omega$. Suppose $\phi \in \Lambda^{1,1}$ is pluriclosed and satisfies:

- $\int_{M} \phi \wedge \phi>0$

- $\int_{M} \phi \wedge \omega>0$

- $\int_{D} \phi>0$ for every irreducible effective divisor with $D \cdot D<0$

Then there exists $f \in C^{\infty}(M)$ such that $\phi+\sqrt{-1} \partial \bar{\partial} f>0$.

For the statement of Theorem 5.6 we need some further background. Recall the Bott-Chern cohomology group:

$$
H_{\mathrm{BC}}^{1,1}=\frac{\left\{\operatorname{Ker} d: \Lambda_{\mathbb{R}}^{1,1} \rightarrow \Lambda_{\mathbb{R}}^{3}\right\}}{i \partial \bar{\partial} \Lambda_{\mathbb{R}}^{0}}
$$

Also, define the groups:

$$
\begin{aligned}
B_{\mathbb{R}}^{1,1} & =d\left\{\Lambda_{\mathbb{R}}^{1}\right\} \cap \Lambda_{\mathbb{R}}^{1,1} \\
H_{\mathbb{R}}^{1,1} & =\frac{\left\{\operatorname{Ker} d: \Lambda_{\mathbb{R}}^{1,1} \rightarrow \Lambda_{\mathbb{R}}^{3}\right\}}{B_{\mathbb{R}}^{1,1}}
\end{aligned}
$$

Lemma 5.5 Let $\left(M^{4}, \omega, J\right)$ be a complex surface with pluriclosed metric. Then there are exact sequences

$$
\begin{gathered}
0 \longrightarrow \frac{B_{\mathbb{R}}^{1,1}}{i \partial \bar{\partial} \Lambda_{\mathbb{R}}^{0}} \longrightarrow H_{B C}^{1,1} \longrightarrow H_{\mathbb{R}}^{1,1} \longrightarrow 0 \\
0 \longrightarrow i \partial \bar{\partial} \Lambda_{\mathbb{R}}^{0} \longrightarrow B_{\mathbb{R}}^{1,1} \longrightarrow \mathbb{R}
\end{gathered}
$$

where the final map above is given by the $L^{2}$ inner product with $\omega$.

Proof We include the elementary proof for convenience. The first exact sequence is tautological. For the second sequence, fix $\mu \in B_{\mathbb{R}}^{1,1}$ satisfying

$$
\int_{M} \mu \wedge \omega=0
$$


It follows from the maximum principle that the adjoint of $\operatorname{tr}_{\omega} \partial \bar{\partial}$ has kernel only constant functions since the adjoint operator annihilates constants. Thus by standard theory we can now solve $\Delta u=\operatorname{tr}_{\omega} \mu$. Thus $i \partial \bar{\partial} u-\mu$ is exact, and also anti-self-dual since its inner product with $\omega$ vanishes. Thus it vanishes, and the lemma follows.

Furthermore (see Teleman [30, Lemma 2.3]), if $b_{1}(M)$ is odd, the space

$$
\Gamma=\frac{B_{\mathbb{R}}^{1,1}}{i \partial \bar{\partial} \Lambda_{\mathbb{R}}^{0}}
$$

is identified with $\mathbb{R}$ via the $L^{2}$ inner product with $\omega$. Let $\gamma_{0}$ denote a positive generator of $\Gamma$. Since the space of pluriclosed metrics on $M$ is connected, this orientation of $\Gamma$ is well-defined.

Theorem 5.6 Let $\left(M^{4}, J\right)$ be a complex non-Kähler surface. Suppose $\phi \in \Lambda^{1,1}$ is pluriclosed. Then $[\phi] \in \mathcal{P}_{\partial+\bar{\partial}}$ if and only if:

- $\int_{M} \phi \wedge \gamma_{0}>0$

- $\int_{D} \phi>0$ for every effective divisor with negative self-intersection

Proof Suppose $\phi \notin \mathcal{P}_{\partial+\bar{\partial}}$. Since the image of $\partial+\bar{\partial}$ is closed in $\Lambda_{\mathbb{R}}^{1,1} \otimes L^{2}(M)$ [4, Lemma 1], one may apply the Hahn-Banach Theorem to conclude the existence of a positive closed current $P$ such that $P(\phi) \leq 0$. We claim that the current $P$ is represented by a convex linear combination of $\left[\gamma_{0}\right]$ and irreducible effective divisors of negative self-intersection. This is [30, Corollary 3.6], and we include a sketch of the proof for convenience. First we note that using arguments from complex analysis one can show that the set of irreducible effective divisors of negative self-intersection is finite [30, Remark 3.3]. Let $\mathcal{C}$ denote the cone generated by $\gamma_{0}$ and this finite set in $H_{B C}^{1,1}$. If $P \notin \mathcal{C}$, there exists a linear hyperplane separating $P$ from $\mathcal{C}$. Specifically we can find an element of the dual space, represented by pairing against a pluriclosed form $\psi$, such that:

$$
\int_{M} \psi \wedge \gamma_{0}>0, \quad \int_{D} \psi>0, \quad P(\psi)=\int_{M} \psi \wedge P<0 .
$$

One can show by direct inspection that $\psi+t \gamma_{0}$ satisfies the criteria of Theorem 5.4, hence there is $f$ such that $\psi+i \partial \bar{\partial} f>0$, and so since $P$ is positive, $P(\psi) \geq 0$, a contradiction.

The following proposition shows that Conjecture 5.2 implies long-time existence of solutions to (1-1) on minimal Class VII surfaces. 
Proposition 5.7 Let $\left(M^{4}, \omega_{0}, J\right)$ be a minimal Class VII surface with pluriclosed metric. Then for all $t \geq 0$ :

$$
\left[\omega_{0}-t c_{1}(\rho)\right] \in \mathcal{P}_{\partial+\bar{\partial}}
$$

Proof By the above theorem it suffices to show the integral inequalities of Theorem 5.6 for $\omega-t c_{1}(\rho), t$ arbitrary. Since $\gamma_{0}$ is exact the first inequality is trivial. Also, for any effective divisor $D$ we know that $\int_{M} c_{1}(D) \wedge c_{1} \leq 0$, hence

$$
\int_{D} \omega-t c_{1} \geq \int_{D} \omega>0 \text {. }
$$

The result follows.

Let us furthermore describe how we expect the presence of rational curves to enter into the singularity formation of solutions to (1-1) on Class VII surfaces. As can be seen by elementary calculations of the evolution of the degree, one has that solutions to (1-1) on Class VII surfaces have volume growing at least quadratically in time. Furthermore, the area of divisors will grow as $K \cdot D$, where $K$ is the canonical class. This pairing is always nonnegative, and is zero on rational curves. Thus, if we renormalize to fix the volume, the boundary of the cone $\mathcal{P}_{\partial+\bar{\partial}}$ should be reached by collapsing a curve. This is made clearer in Section 7 where we examine nonsingular solutions. One can observe at this point though that according to our characterization of $\mathcal{P}_{\partial+\bar{\partial}}$ in Theorem 5.6, the boundary may be reached, after volume normalizing, by having $\lim _{t \rightarrow \infty} \int_{M} \omega(t) \wedge \gamma_{0}=0$. In other words, perhaps it is this condition that fails, and not the presence of a curve satisfying $K \cdot D=0$. The following proposition effectively negates this possibility.

Proposition 5.8 Let $\left(M^{4}, J\right)$ be a complex non-Kähler surface. Suppose $\phi \in \Lambda^{1,1}$ is pluriclosed, and satisfies:

- $\int_{M} \phi \wedge \phi>0$

- $\int_{M} \phi \wedge \gamma_{0} \geq 0$

- $\int_{D} \phi>0$ for every effective divisor with negative self-intersection

Then $\phi \in \mathcal{P}_{\partial+\bar{\partial}}$.

Proof Fix $\tilde{\omega}$ a pluriclosed metric on $M$. Note that $\phi \in \mathcal{P}_{\partial+\bar{\partial}}$ if and only if $\psi:=$ $\phi+a \gamma_{0} \in \mathcal{P}_{\partial+\bar{\partial}}$. Now observe that, for $a>0$ :

$$
\int_{M} \psi \wedge \psi=\int_{M} \phi \wedge \phi+2 a \int_{M} \phi \wedge \gamma_{0}>0
$$


Also, since $\int_{M} \tilde{\omega} \wedge \gamma_{0}>0$, we may choose $a$ large enough so that

$$
\int_{M} \psi \wedge \tilde{\omega}=\int_{M} \phi \wedge \tilde{\omega}+a \int_{M} \gamma_{0} \wedge \tilde{\omega}>0
$$

Finally, since $\gamma_{0}$ is $d$-exact

$$
\int_{D} \psi=\int_{D} \phi>0
$$

Therefore $\psi$ satisfies all three conditions of Buchdahl's positivity criterion [5, page 1533], so there is a function $f$ such that $\psi+i \partial \bar{\partial} f>0$. The proposition follows.

In light of this proposition, we make a final conjecture, specializing Conjecture 5.3 to the case $n=2$. Note that every pluriclosed metric satisfies $\int_{M} \phi \wedge \gamma_{0}>0$, so the second condition of Proposition 5.8 is automatically satisfied at any potentially singular time for a solution to (1-1).

Conjecture 5.9 (Strong existence conjecture for surfaces) Let $\left(M^{4}, g_{0}, J\right)$ be a compact complex surface with pluriclosed metric. Let $\omega(t)$ be the solution to (1-1) with initial condition $\omega_{0}$. Suppose $\omega(t)$ exists on $[0, \tau)$ and that:

- $\lim _{t \rightarrow \tau} \int_{M} \omega \wedge \omega>0$.

- There exists $A>0$ so that

$$
\frac{1}{A}<\lim _{t \rightarrow \tau} \int_{D} \omega<A
$$

for every effective divisor with negative self-intersection.

Then there exists a uniform bound on the curvature of $\omega(t)$ depending on $A$, and moreover the curvature remains bounded after the diameter is rescaled to unit size.

Furthermore, suppose $\omega(t)$ is the solution to volume-normalized pluriclosed flow with initial condition $\omega_{0}$. Suppose $\omega(t)$ exists on $[0, \tau)$ and that there exists $A>0$ so that

$$
\lim _{t \rightarrow \tau} \frac{1}{A}<\int_{D} \omega<A
$$

for every effective divisor with negative self-intersection. Then there exists a uniform bound on the curvature of $\omega(t)$ depending on $A$, and moreover the curvature remains bounded after the diameter is rescaled to unit size. 
Next we want to exploit this cohomology picture to reduce solutions to (1-1) to an equation on a one-form. We show that under certain cohomological conditions related to the Frölicher spectral sequence, solutions to Equation (5-1) automatically reduce to solutions to a certain equation on one-forms defined below. These cohomological conditions are automatically satisfied in the case of complex surfaces. We start with some preliminary lemmas.

Lemma 5.10 Let $\left(M^{4}, J\right)$ be a complex surface. Then the map

$$
\text { ว: } H^{1}\left(\Omega^{1}\right) \rightarrow H^{1}\left(\Omega^{2}\right)
$$

is the zero map.

Proof This argument is adapted from arguments in Barth, Hulek, Peters and Van de Ven [2, IV Section 2]. Let $\mathcal{S}$ denote the sheaf of closed holomorphic 1-forms on $M$. There is an exact sequence of sheaves:

$$
0 \longrightarrow \mathbb{C}_{M} \longrightarrow \mathcal{O}_{M} \stackrel{d}{\longrightarrow} \mathcal{S} \longrightarrow 0
$$

Since holomorphic forms on complex surfaces are closed, there is an exact sequence of cohomology groups:

$$
0 \longrightarrow H^{0}\left(\Omega^{1}\right) \longrightarrow H^{1}(M, \mathbb{C}) \longrightarrow H^{1}\left(\mathcal{O}_{M}\right) \stackrel{\partial}{\longrightarrow} H^{1}\left(\Omega^{1}\right)
$$

It follows from the signature theorem and the Riemann-Roch formula (see [2, Theorem IV 2.7]) that $b_{1}=h^{1,0}+h^{0,1}$. Therefore the third map above is surjective, and hence the last map is the zero map. Applying Stokes' Theorem and Serre duality we can conclude that $\partial: H^{1}\left(\Omega^{1}\right) \rightarrow H^{1}\left(\Omega^{2}\right)$ is also the zero map.

Lemma 5.11 Let $\left(M^{4}, J\right)$ be a complex surface and suppose $h^{0,2}=0$. Let $\alpha \in \Lambda^{2,1}$ be a $d$-exact $(2,1)$-form. Then there exists $\gamma \in \Lambda^{2,0}$ such that $\alpha=\bar{\partial} \gamma$.

Proof Since by assumption $\alpha$ is exact, we conclude that

$$
\alpha=d \beta=d\left(\beta^{2,0}+\beta^{1,1}+\beta^{0,2}\right)=\bar{\partial} \beta^{2,0}+\partial \beta^{1,1}+\bar{\partial} \beta^{1,1}+\partial \beta^{0,2},
$$

where the remaining terms vanish for dimensional reasons. Decomposing this equation into types yields the two equations:

$$
\begin{aligned}
\alpha & =\bar{\partial} \beta^{2,0}+\partial \beta^{1,1}, \\
0 & =\bar{\partial} \beta^{1,1}+\partial \beta^{0,2} .
\end{aligned}
$$


Note that $\beta^{0,2}$ defines a class in $H^{0,2}(M)$. Since $h^{0,2}=0$, there exists $\mu^{0,1}$ such that $\beta^{0,2}=\bar{\partial} \mu$. We therefore conclude that

$$
0=\bar{\partial}\left(\beta^{1,1}-\partial \mu^{0,1}\right)
$$

Therefore $\beta^{1,1}-\partial \mu^{0,1}$ defines a class in $H^{1,1}(M)$. Now, by Lemma 5.10 we know that $\partial: H^{1}\left(\Omega^{1}\right) \rightarrow H^{1}\left(\Omega^{2}\right)$ is the zero map. Therefore $\partial g\left(\beta^{1,1}-\partial \mu^{0,1} g\right)=\partial \beta^{1,1}$ represents the zero class in $H^{1}\left(\Omega^{2}\right) \cong H^{2,1}(M)$. Therefore there exists $\rho^{2,0}$ such that $\partial \beta^{1,1}=\bar{\partial} \rho^{2,0}$. Plugging this back into the above equation yields

$$
\alpha=\bar{\partial} \beta^{2,0}+\bar{\partial} \rho^{2,0}
$$

and the result follows.

Lemma 5.12 Let $\left(M^{4}, J\right)$ be a complex surface. Then:

$$
\operatorname{Ker}\left\{\bar{\partial}: \Lambda^{2,0} \rightarrow \Lambda^{2,1}\right\} \cap \operatorname{Im}\left\{\partial: \Lambda^{1,0} \rightarrow \Lambda^{2,0}\right\}=\{0\}
$$

Proof Let $\phi=\partial \alpha, \bar{\partial} \phi=0, \alpha \in \Lambda^{1,0}$. A general calculation for complex surfaces shows that for any metric $g,|\phi|^{2} d V_{g}=\phi \wedge \bar{\phi}$. Thus

$$
\|\phi\|_{L^{2}}^{2}=\int_{M} \phi \wedge \bar{\phi}=\int_{M} \phi \wedge \bar{\partial} \bar{\alpha}=0
$$

by Stokes' Theorem.

Lemma 5.13 Let $\left(M^{4}, \omega, J\right)$ be a complex surface with pluriclosed metric. Suppose $b_{1}$ is odd and $h^{0,2}=0$. Then $[\partial \omega] \neq 0 \in H^{3}(M, \mathbb{C})$ and $[\partial \omega] \neq 0 \in H^{2,1}(M)$.

Proof Suppose that $\partial \omega$ is a $d$-exact form. By Lemma 5.11 we conclude that $\partial \omega=\bar{\partial} \beta$. Note this also holds trivially if we assume $[\partial \omega]=0 \in H^{2,1}(M)$. Now, $\bar{\beta} \in \Lambda^{0,2}$ obviously satisfies $\bar{\partial} \bar{\beta}=0$. However, since $h^{0,2}=0$ one can write $\bar{\beta}=\bar{\partial} \bar{\alpha}$. Therefore $\partial \omega=\bar{\partial} \partial \alpha$ and, taking conjugates, $\bar{\partial} \omega=\partial \bar{\partial} \bar{\alpha}$. Let $\tilde{\omega}=\omega-\partial \alpha-\bar{\partial} \bar{\alpha}$. One computes directly that

$$
\begin{aligned}
d \widetilde{\omega} & =(\partial+\bar{\partial})(\omega-\partial \alpha-\bar{\partial} \bar{\alpha}) \\
& =\partial \omega-\bar{\partial} \partial \alpha+\bar{\partial} \omega-\partial \bar{\partial} \bar{\alpha} \\
& =0 .
\end{aligned}
$$

Since the $(1,1)$ component of $\tilde{\omega}$ is positive definite, it follows that

$$
\int_{M} \tilde{\omega} \wedge \tilde{\omega}>0
$$

Since $b_{1}$ is odd, the intersection form of $M$ is negative definite [2, Theorem IV.2.14], so this is a contradiction. 
Theorem 5.14 Let $\left(M^{4}, \omega, J\right)$ be a complex surface with pluriclosed metric satisfying $h^{0,1} \leq 1$. Let:

$$
B=\pi c_{1}(\omega)+\partial \beta+\bar{\partial} \bar{\beta} \in \pi c_{1} \in \mathcal{H}_{\partial+\bar{\partial}}
$$

Suppose $\widetilde{\omega}=\omega+\partial \alpha+\bar{\partial} \bar{\alpha}$ is a solution to

$$
\partial \partial_{\widetilde{\omega}}^{*} \widetilde{\omega}+\bar{\partial} \bar{\partial}_{\widetilde{\omega}}^{*} \widetilde{\omega}+\frac{\sqrt{-1}}{2} \partial \bar{\partial} \log \operatorname{det} \widetilde{g}=B
$$

Then

$$
\beta=\partial_{\widetilde{\omega}}^{*} \widetilde{\omega}+\frac{\sqrt{-1}}{4} \bar{\partial} \log \frac{\operatorname{det} \tilde{g}}{\operatorname{det} g}
$$

Proof Taking $\partial$ of Equation (5-1) yields

$$
0=\partial \bar{\partial} \bar{\partial}_{\widetilde{\omega}}^{*} \widetilde{\omega}-\partial \bar{\partial} \bar{\beta}
$$

or equivalently

$$
0=\bar{\partial}\left[\partial \bar{\partial}_{\widetilde{\omega}}^{*} \widetilde{\omega}-\partial \bar{\beta}\right] .
$$

Therefore $\partial \bar{\partial}_{\widetilde{\omega}}^{*} \widetilde{\omega}-\partial \bar{\beta}$ is a $\bar{\partial}$-closed, $\partial$-exact $(2,0)$-form. Using Lemma 5.12 we conclude that $\partial \bar{\partial}_{\widetilde{\omega}}^{*} \widetilde{\omega}-\partial \bar{\beta}=0$. Conjugating yields $\bar{\partial}\left[\partial_{\widetilde{\omega}}^{*} \widetilde{\omega}-\beta\right]=0$. Thus we may write the Hodge decomposition of $\partial_{\widetilde{\omega}}^{*} \widetilde{\omega}-\beta$ with respect to $\Delta_{\bar{\partial}, \omega}$ as

$$
\partial_{\widetilde{\omega}}^{*} \widetilde{\omega}-\beta=h+\bar{\partial} f
$$

where $h \in H^{0,1}$. Next we claim that $h$ vanishes. Once we know this, differentiating and plugging into (5-1) yields $f=-(\sqrt{-1} / 4) \bar{\partial} \log (\operatorname{det} \tilde{g} / \operatorname{det} g)$ and the theorem follows. First of all, if $h^{0,1}=0$ this is trivial, and since this observation holds in general dimension we record this as Proposition 5.15 below. Next suppose $h^{0,1}=1$. We compute:

$$
\int_{M}\left\langle h, \partial_{\omega}^{*} \omega\right\rangle_{\omega} d V=\int_{M}\left\langle\partial_{\widetilde{\omega}}^{*} \tilde{\omega}-\beta-\bar{\partial} f, \partial_{\omega}^{*} \omega\right\rangle_{\omega} d V
$$

First observe

$$
\begin{aligned}
\int_{M}\left\langle\bar{\partial} f, \partial_{\omega}^{*} \omega\right\rangle_{\omega} d V & =\int_{M}\langle\partial \bar{\partial} f, \omega\rangle_{\omega} d V_{g} \\
& =\int_{M} \partial \bar{\partial} f \wedge \omega \\
& =0
\end{aligned}
$$

by Stokes' Theorem, using that $\partial \bar{\partial} \omega=0$. Next we compute

$$
\int_{M}\left\langle-\beta, \partial_{\omega}^{*} \omega\right\rangle_{\omega} d V=\int_{M}\langle-\partial \beta, \omega\rangle_{\omega} d V=\int_{M}\left\langle-\frac{1}{2}(\partial \beta+\bar{\partial} \bar{\beta}), \omega\right\rangle
$$


since $\omega$ is real. Also we compute using (5-1):

$$
\begin{aligned}
\int_{M}\left\langle\partial_{\widetilde{\omega}}^{*} \widetilde{\omega}, \partial_{\omega}^{*} \omega\right\rangle_{\omega} d V & =\int_{M}\left\langle\partial \partial_{\widetilde{\omega}}^{*} \widetilde{\omega}, \omega\right\rangle_{\omega} d V \\
& =\int_{M}\left\langle\frac{1}{2}\left(\partial \partial_{\widetilde{\omega}}^{*} \widetilde{\omega}+\bar{\partial} \bar{\partial}_{\widetilde{\omega}}^{*} \widetilde{\omega}\right), \omega\right\rangle_{\omega} d V \\
& =\int_{M}\left\langle\frac{1}{2}\left(c_{1}(\widetilde{\omega})-c_{1}(\omega)+\partial \beta+\bar{\partial} \bar{\beta}\right), \omega\right\rangle_{\omega} d V \\
& =\int_{M}\left\langle\frac{1}{2}(\partial \beta+\bar{\partial} \bar{\beta}), \omega\right\rangle_{\omega} d V
\end{aligned}
$$

where in the last line we used that $c_{1}(\widetilde{\omega})-c_{1}(\omega)=\partial \bar{\partial} \phi$ and used that $\omega$ is orthogonal to the image of $\partial \bar{\partial}$ since it is pluriclosed. It follows that

$$
\int_{M}\left\langle h, \partial_{\omega}^{*} \omega\right\rangle_{\omega} d V=0 .
$$

However, by Lemma 5.13, $[\partial \omega] \neq 0$, and $h^{2,1}=1$ by Serre duality, therefore there is a nonzero constant $a$ such that $[\partial \omega]=[a * h]$ (since $h$ is $\bar{\partial}^{*}$-closed, $* h$ defines a cohomology class). Thus

$$
0=\int_{M}\left\langle h, \partial_{\omega}^{*} \omega\right\rangle=\int_{M}\langle * h, \partial \omega\rangle=a \int_{M}|h|^{2} .
$$

Therefore, $h=0$.

Proposition 5.15 Let $\left(M^{2 n}, \omega, J\right)$ be a complex manifold with pluriclosed metric satisfying $h^{0,1}=0$ and

$$
\operatorname{Ker}\left\{\bar{\partial}: \Lambda^{2,0} \rightarrow \Lambda^{2,1}\right\} \cap \operatorname{Im}\left\{\partial: \Lambda^{1,0} \rightarrow \Lambda^{2,0}\right\}=\{0\} .
$$

Let:

$$
B=\pi c_{1}(\omega)+\partial \beta+\bar{\partial} \bar{\beta} \in \pi c_{1} \in \mathcal{H}_{\partial+\bar{\partial}}
$$

Suppose $\widetilde{\omega}=\omega+\partial \alpha+\bar{\partial} \bar{\alpha}$ is a solution to

$$
\Phi(\widetilde{\omega})=B .
$$

Then:

$$
\beta=\partial_{\widetilde{\omega}}^{*} \widetilde{\omega}+\frac{\sqrt{-1}}{4} \bar{\partial} \log \frac{\operatorname{det} \tilde{g}}{\operatorname{det} g}
$$

Proof The proof is clear from the proof of Theorem 5.14. 
Theorem 5.14 may be applied to reduce solutions to (1-1) on surfaces.

Theorem 5.16 Let $\left(M^{4}, g_{0}, J\right)$ be a pluriclosed surface. Say the solution to (1-1) with initial condition $g_{0}$ exists on a maximal time interval $[0, T), T<\infty$. Fix a background metric $\rho$ and express

$$
\omega(t)=\omega_{0}+\partial \alpha(t)+\bar{\partial} \bar{\alpha}(t)-t c_{1}(\rho) .
$$

Then $\alpha$ satisfies:

$$
\frac{\partial}{\partial t} \alpha=\partial_{\omega}^{*} \omega+\frac{\sqrt{-1}}{4} \bar{\partial} \log \frac{\operatorname{det} \tilde{g}}{\operatorname{det} g}
$$

Proof We differentiate the expression for $\omega(t)$ and use equation (1-1) to compute:

$$
\partial \dot{\alpha}+\bar{\partial} \dot{\bar{\alpha}}-c_{1}(\rho)=-\Phi(\omega)
$$

One may apply Theorem 5.14 with $\beta=\dot{\alpha}$ to conclude the result.

Thus we have canonically reduced solutions to (1-1) to solutions of this equation, coupled to an ODE. There is a natural gauge to equation (1-5).

Definition 5.17 Given $\left(M^{2 n}, g, J\right)$ a complex manifold with Hermitian metric $g$, let $\alpha \in \Lambda^{0,1}$ and let $\psi \in \Lambda^{1,1}, d \psi=0$. Let:

$$
[\alpha, \psi]=\left\{(\alpha+\beta, \psi-\partial \beta-\bar{\partial} \bar{\beta}) \mid \beta \in \Lambda^{0,1}, \bar{\partial} \beta=0\right\}
$$

We will refer to $[\alpha, \psi]$ as the gauge equivalence class of $(\alpha, \psi)$.

Proposition 5.18 Let $(\alpha(t), \psi(t))$ be a solution to (1-5). Then $(\alpha(t), \psi(t))$ is gaugeequivalent to a pair $(\widetilde{\alpha}(t), \widetilde{\psi}(t))$ such that $\widetilde{\alpha}(t)$ solves a parabolic equation.

Proof We find a gauge near time $t=0$ for which $\tilde{\alpha}(t)$ solves a parabolic equation. We take the $\partial$-Hodge decomposition of $\alpha(t)$ with respect to $\omega_{0}$. Specifically, consider

$$
\alpha(t)=\bar{\partial}_{\omega_{0}}^{*} v(t)+\phi(t)+\bar{\partial} f(t),
$$

where $\bar{\partial} \phi(t)=\bar{\partial}_{\omega_{0}}^{*} \phi=0$. Define

$$
\begin{aligned}
\tilde{\alpha}(t) & =\alpha(t)-\phi(0)-\bar{\partial} f(0), \\
\tilde{\psi}(t) & =\psi(t)-\partial(\phi(0)+\bar{\partial} f(0))-\bar{\partial}(\bar{\phi}(0)+\partial \bar{f}(0)) .
\end{aligned}
$$

Note that by construction $\bar{\partial}_{\omega_{0}}^{*} \widetilde{\alpha}(t)=0$. Also, note that $\widetilde{\omega}=\omega_{0}+\partial \widetilde{\alpha}+\bar{\partial} \overline{\widetilde{\alpha}}+\tilde{\psi}$ by construction as well. Furthermore, we have that:

$$
\frac{\partial}{\partial t} \widetilde{\alpha}_{\mid t=0}=\partial_{\omega_{0}}^{*} \omega_{0}+\frac{\sqrt{-1}}{4} \bar{\partial} \log \frac{\omega_{0}^{n}}{\rho^{n}}
$$


Our aim is to show that the right hand side is an elliptic operator for $\alpha$. For a given metric $\omega$ we have the general coordinate formula:

$$
\left(\partial_{\omega}^{*} \omega\right)_{\bar{j}}=\frac{\sqrt{-1}}{2} g^{p \bar{q}}\left(\partial_{\bar{q}} g_{p \bar{j}}-\partial_{\bar{j}} g_{p \bar{q}}\right)
$$

Likewise we have:

$$
\left(\frac{\sqrt{-1}}{4} \bar{\partial} \log \frac{\omega^{n}}{\rho^{n}}\right)_{\bar{j}}=\frac{\sqrt{-1}}{4}\left(g^{p \bar{q}} \partial_{\bar{j}} g_{p \bar{q}}-\rho^{p \bar{q}} \partial_{j} \rho_{p \bar{q}}\right)
$$

Specializing these two formulas to the case $\widetilde{\omega}=\omega+\partial \widetilde{\alpha}+\bar{\partial} \overline{\widetilde{\alpha}}+\widetilde{\psi}$, we compute:

$$
\begin{aligned}
\left(\partial_{\widetilde{\omega}}^{*} \widetilde{\omega}+\frac{\sqrt{-1}}{4} \bar{\partial} \log \frac{\widetilde{\omega}^{n}}{\rho^{n}}\right)_{\bar{j}} & \\
= & \frac{\sqrt{-1}}{2} \widetilde{g}^{p \bar{q}}\left(\partial_{\bar{q}}\left(\partial_{p} \widetilde{\alpha}_{\bar{j}}+\partial_{\bar{j}} \widetilde{\alpha}_{p}\right)-\partial_{\bar{j}}\left(\partial_{p} \widetilde{\alpha}_{\bar{q}}+\partial_{\bar{q}} \widetilde{\alpha}_{p}\right)\right) \\
& +\frac{\sqrt{-1}}{4} \widetilde{g}^{p \bar{q}}\left(\partial_{\bar{j}}\left(\partial_{p} \widetilde{\alpha}_{\bar{q}}+\partial_{\bar{q}} \widetilde{\alpha}_{p}\right)\right)+\mathcal{O}(\partial \widetilde{\alpha}) \\
= & \frac{\sqrt{-1}}{2} \widetilde{g}^{p \bar{q}} \partial_{p} \partial_{\bar{q}} \widetilde{\alpha}_{\bar{j}}+\frac{\sqrt{-1}}{4} \widetilde{g}^{p \bar{q}} \partial_{\bar{j}} \partial_{\bar{q}} \widetilde{\alpha}_{p}-\frac{\sqrt{-1}}{4} \widetilde{g}^{p \bar{q}} \partial_{\bar{j}} \partial_{p} \widetilde{\alpha}_{\bar{q}}
\end{aligned}
$$

Now, using the condition $\bar{\partial}_{\omega_{0}}^{*} \tilde{\alpha}=0$, we compute that

$$
0=\partial_{\bar{j}} \bar{\partial}_{\omega_{0}}^{*} \tilde{\alpha}=g^{p \bar{q}} \partial_{\bar{j}} \partial_{p} \widetilde{\alpha}_{\bar{q}}+\mathcal{O}(\partial \widetilde{\alpha}) .
$$

Likewise since $\partial_{\omega_{0}}^{*} \overline{\widetilde{\alpha}}=0$ we have $g^{p \bar{q}} \partial_{\bar{j}} \partial_{\bar{q}} \widetilde{\alpha}_{p}=\mathcal{O}(\partial \widetilde{\alpha})$. Since $\tilde{g}(0)=g_{0}$ we conclude that:

$$
\left(\partial_{\widetilde{\omega}}^{*} \widetilde{\omega}+\frac{\sqrt{-1}}{4} \bar{\partial} \log \frac{\widetilde{\omega}^{n}}{\rho^{n}}\right)_{\bar{j}}(0)=\frac{\sqrt{-1}}{2} \tilde{g}^{p \bar{q}} \partial_{p} \partial_{\bar{q}} \tilde{\alpha}_{\bar{j}}
$$

which is a strictly elliptic operator. The result follows.

\section{Pluriclosed flow as a gradient flow}

In this section we exhibit that (1-1) is the gradient flow of the first eigenvalue of a certain Schrödinger operator associated to the time-dependent metric. What we actually show is that, after pulling back a solution to (1-1) by the one-parameter family of diffeomorphisms generated by the vector field dual to the Lee form, one produces a solution to the renormalization group flow of a nonlinear sigma model arising in string theory (see Polchinski [24, 108-112]). This surprising fact both exhibits a connection between pluriclosed flow and mathematical physics, and from another point of view produces a large class of interesting examples of the renormalization group flow. 
Let us recall some notation from the introduction. Fix $\left(M^{2 n}, g, J\right)$ a complex manifold with pluriclosed metric. Let $\nabla$ denote the Bismut connection, Rc the Ricci tensor of the Bismut connection, $\mathrm{Rc}^{g}$ the Ricci curvature of $g, T$ the torsion of $\nabla$, and

$$
\theta=-J d^{*} \omega
$$

the Lee form of $\omega$. Lastly, as in the introduction, let $P$ denote the representative of $c_{1}(M, J)$ associated to the Bismut connection $\nabla$. We need to show some identities relating these tensors. We start by recording some basic calculations which appear in Ivanov and Papadopoulos [16]. It is important to remember below that Rc is not symmetric, and $P$ is not $(1,1)$.

Lemma 6.1 [16, Proposition 3.1] Let $\left(M^{2 n}, g, J\right)$ be a pluriclosed structure. Then:

$$
\begin{aligned}
\operatorname{Rc}^{g}(X, Y) & =\operatorname{Rc}(X, Y)+\frac{1}{2} d^{*} T(X, Y)+\frac{1}{4} \sum_{i=1}^{2 n} g\left(T\left(X, e_{i}\right), T\left(Y, e_{i}\right)\right) \\
P(X, Y) & =\operatorname{Ric}(X, J Y)+\nabla_{X} \theta(J Y)
\end{aligned}
$$

$\operatorname{Rc}(Y, J X)+\operatorname{Rc}(X, J Y)=-\left(\nabla_{X} \theta\right)(J Y)-\nabla_{Y}(\theta)(J X)$

$$
P(J X, J Y)-P(X, Y)=d^{*} T(J X, Y)-d^{\nabla} \theta(J X, Y)
$$

where $d^{\nabla}$ is the exterior derivative induced by $\nabla$.

Proof Note that the tensor $\lambda$ from [16] vanishes when $\partial \bar{\partial} \omega=0$. The third line is $[16$, (3.9)].

Let $H(X, Y):=P^{1,1}(J X, Y)$. In particular, note that (1-1) is equivalent to:

$$
\frac{\partial}{\partial t} g=-H
$$

Lemma 6.2 Let $\left(M^{2 n}, g, J\right)$ be a pluriclosed structure. Then:

$$
H(X, Y)=\frac{1}{2}\left[\operatorname{Rc}(X, Y)+\operatorname{Rc}(J X, J Y)+\nabla_{X} \theta(Y)+\nabla_{J X} \theta(J Y)\right]
$$

Proof We directly compute using Lemma 6.1:

$$
\begin{aligned}
H(X, Y) & =P^{1,1}(J X, Y) \\
& =\frac{1}{2}[P(J X, Y)+P(J J X, J Y)] \\
& =\frac{1}{2}[P(J X, Y)-P(X, J Y)] \\
& =\frac{1}{2}\left[\operatorname{Ric}(J X, J Y)+\left(\nabla_{J X} \theta\right)(J Y)-\operatorname{Ric}(X, J J Y)-\left(\nabla_{X} \theta\right)(J J Y)\right] \\
& =\frac{1}{2}\left[\operatorname{Ric}(X, Y)+\operatorname{Ric}(J X, J Y)+\left(\nabla_{X} \theta\right)(Y)+\left(\nabla_{J X} \theta\right)(J Y)\right]
\end{aligned}
$$


Proposition 6.3 Given $\left(M^{2 n}, \omega(t), J\right)$ a solution to (1-1), one has

$$
\frac{\partial}{\partial t} g=\left[-\mathrm{Rc}^{g}+\frac{1}{4} \sum_{i=1}^{2 n} g\left(T\left(X, e_{i}\right), T\left(Y, e_{i}\right)\right)-\frac{1}{2} \mathcal{L}_{\theta^{\sharp}} g\right]
$$

where $\theta^{\sharp}$ is the vector field dual to $\theta$, taken with respect to the time varying metric.

Proof Using the third line of Lemma 6.1, we compute:

$$
\begin{aligned}
\operatorname{Ric}(J X, J Y)+\left(\nabla_{J X} \theta\right)(J Y) & =-\operatorname{Ric}(Y, J J X)-\left(\nabla_{Y} \theta\right)(J J X) \\
& =\operatorname{Ric}(Y, X)+\left(\nabla_{Y} \theta\right)(X)
\end{aligned}
$$

Plugging this into Lemma 6.2 yields:

$$
2 H(X, Y)=\operatorname{Ric}(X, Y)+\operatorname{Ric}(Y, X)+\left(\nabla_{X} \theta\right)(Y)+\left(\nabla_{Y} \theta\right)(X)
$$

The first two terms are twice the symmetric part of Ric, which is easily computed from the first line of Lemma 6.1. It remains to show that the last two terms are $\mathcal{L}_{\theta \sharp} g$. To do this we compute in coordinates, if $\Gamma$ denotes the connection coefficients of the Bismut connection and $\Gamma^{L C}$ the Levi-Civita connection,

$$
\nabla_{i} \theta_{j}=\partial_{i} \theta_{j}-\Gamma_{i j}^{k} \theta_{k}=\partial_{i} \theta_{j}-\left(\Gamma^{L C}+\frac{1}{2} T\right)_{i j}^{k} \theta_{k}=D_{i} \theta_{j}-T_{i j}^{k} \theta_{k},
$$

where of course $D$ denotes the Levi-Civita derivative. But $T$ is totally skew, thus:

$$
\nabla_{i} \theta_{j}+\nabla_{j} \theta_{i}=D_{i} \theta_{j}+D_{j} \theta_{i}-\frac{1}{2} T_{i j}^{k} \theta_{k}-\frac{1}{2} T_{j i}^{k} \theta_{k}=D_{i} \theta_{j}+D_{j} \theta_{i}=\left(\mathcal{L}_{\theta^{\sharp}} g\right)_{i j}
$$

Proposition 6.4 Given $\left(M^{2 n}, \omega(t), J\right)$ a solution to (1-1), one has:

$$
\frac{\partial}{\partial t} T=\frac{1}{2}\left[\Delta_{L B, g(t)} T-\mathcal{L}_{\theta^{\sharp}} T\right]
$$

Proof Recall that $T=d^{c} \omega$, where $d^{c}=i(\bar{\partial}-\partial)$. Therefore:

$$
\frac{\partial}{\partial t} T=\frac{\partial}{\partial t} d^{c} \omega=-d^{c}\left(P^{1,1}\right)
$$

Now note that, since $P$ is closed:

$$
\begin{aligned}
0 & =d P \\
& =(\partial+\bar{\partial})\left(P^{1,1}+P^{2,0}+P^{0,2}\right) \\
& =\partial P^{1,1}+\bar{\partial} P^{1,1}+\partial P^{2,0}+\bar{\partial} P^{2,0}+\partial P^{0,2}+\bar{\partial} P^{0,2}
\end{aligned}
$$


By examining types we conclude from this the equations:

$$
\begin{aligned}
0 & =\partial P^{2,0}=\bar{\partial} P^{0,2} \\
\partial P^{1,1} & =-\bar{\partial} P^{2,0} \\
\bar{\partial} P^{1,1} & =-\partial P^{0,2}
\end{aligned}
$$

It follows that

$$
-d^{c}\left(P^{1,1}\right)=-i(\bar{\partial}-\partial)\left(P^{1,1}\right)=i \partial P^{0,2}-i \bar{\partial} P^{2,0} .
$$

For convenience, set $\psi=d^{*} T-d^{\nabla} \theta$. Now fix local complex coordinates, and compute using the last line of Lemma 6.1:

$$
\begin{aligned}
\left(i \partial P^{0,2}\right)_{i \bar{j} \bar{k}} & =-\frac{i}{2} \partial_{i}\left[d^{*} T\left(J \partial_{\bar{j}}, \partial_{\bar{k}}\right)-d^{\nabla} \theta\left(J \partial_{\bar{j}}, \partial_{\bar{k}}\right)\right] \\
& =-\frac{1}{2} \partial_{i}\left[d^{*} T\left(\partial_{\bar{j}}, \partial_{\bar{k}}\right)-d^{\nabla} \theta\left(\partial_{\bar{j}}, \partial_{\bar{k}}\right)\right]=-\frac{1}{2}\left(\partial \psi^{0,2}\right)_{i \bar{j} \bar{k}}
\end{aligned}
$$

Likewise we can compute:

$$
\begin{aligned}
\left(-i \bar{\partial} P^{2,0}\right)_{\bar{i} j k} & =\frac{i}{2} \partial_{\bar{i}}\left[d^{*} T\left(J \partial_{j}, \partial_{k}\right)-d^{\nabla} \theta\left(J \partial_{j}, \partial_{k}\right)\right] \\
& =-\frac{1}{2} \partial_{\bar{i}}\left[d^{*} T\left(\partial_{j}, \partial_{k}\right)-d^{\nabla} \theta\left(\partial_{j}, \partial_{k}\right)\right]=-\frac{1}{2}\left(\bar{\partial} \psi^{2,0}\right)_{\bar{i} j k}
\end{aligned}
$$

Note that it is a consequence of the last line of Lemma 6.1 that $\psi^{1,1}=0$. In particular, we have $\frac{1}{2} \psi=\rho^{2,0}+\rho^{0,2}$ and so

$$
\partial \psi^{2,0}=\bar{\partial} \psi^{0,2}=0 .
$$

Collecting these calculations yields

$$
-d^{c} P^{1,1}=-\frac{1}{2} d \psi=-\frac{1}{2}\left(d d^{*} T-d d^{\nabla} \theta\right) .
$$

Since $T$ is closed, it follows that $d d^{*} T=-\Delta_{L B, g(t)} T$. Finally, we observe a formula for $d^{\nabla} \theta$ :

$$
\begin{aligned}
\left(d^{\nabla} \theta\right)_{i j} & =\nabla_{i} \theta_{j}-\nabla_{j} \theta_{i} \\
& \left.=\partial_{i} \theta_{j}-\left(\Gamma^{L C}+\frac{1}{2} T\right)_{i j}^{k} \theta_{k}-\partial_{j} \theta_{i}+\left(\Gamma^{L C}+\frac{1}{2} T\right)_{j i}^{k} \theta_{k}=\left(d \theta-\theta^{\#}\right\lrcorner T\right)_{i j}
\end{aligned}
$$

It follows from the Cartan formula and the fact that $T$ is closed that:

$$
\begin{aligned}
d d^{\nabla} \theta & \left.=d\left(d \theta-\theta^{\sharp}\right\lrcorner T\right) \\
& \left.=-d\left(\theta^{\sharp}\right\lrcorner T\right) \\
& \left.=-\mathcal{L}_{\theta^{\sharp}} T+\theta^{\sharp}\right\lrcorner(d T)=-\mathcal{L}_{\theta^{\sharp}} T
\end{aligned}
$$

Therefore $-d^{c} P^{1,1}=\frac{1}{2}\left[\Delta_{L B, g(t)}-\mathcal{L}_{\theta^{\sharp}} T\right]$. 
Theorem 6.5 Let $\left(M^{2 n}, \widetilde{\omega}(t), J\right)$ be a solution to (1-1). Let $X(t)=\frac{1}{2} \widetilde{\theta}^{\sharp}$, where $\sharp$ means the vector dual taken with respect to the time-varying metric, and let $\phi_{t}$ denote the one-parameter family of diffeomorphisms generated by $X(t)$. Let $\tilde{T}$ denote the torsion of the time-varying Bismut connections. Let $\left.(g(t), T(t))=\left(\phi^{*}(\widetilde{g})(t)\right), \phi_{t}^{*}(\widetilde{T})(t)\right)$. Then

$$
\begin{aligned}
\frac{\partial}{\partial t} g & =-\mathrm{Rc}^{g}+\frac{1}{4} \mathcal{H}, \\
\frac{\partial}{\partial t} T & =\frac{1}{2} \Delta_{L B} T,
\end{aligned}
$$

where $\mathcal{H}_{i j}=g^{k l} g^{m n} T_{i k m} T_{j l n}$.

Proof This follows from a standard calculation using Propositions 6.3 and 6.4.

As noted above, the system of equations (6-2) arises naturally in physics as the renormalization group flow of a nonlinear sigma model. By extending Perelman's energy functional [22] to this coupled system, Oliynyk, Suneeta and Woolgar showed that (6-2) is the gradient flow of a nonlinear Schrödinger operator [21]. To discuss this let us generalize the notation slightly. As in the introduction, let $\left(M^{n}, g\right)$ be a Riemannian manifold, and let $T$ denote a three-form on $M$. Let

$$
\mathcal{F}(g, T, f)=\int_{M}\left[R-\frac{1}{12}|T|^{2}+|\nabla f|^{2}\right] e^{-f} d V .
$$

Furthermore set

$$
\lambda(g, T)=\inf _{\left\{f \mid \int_{M} e^{-f} d V=1\right\}} \mathcal{F}(g, T, f) .
$$

Proposition 6.6 (e[21, Proposition 3.1]) The gradient flow of $\lambda$ is

$$
\begin{aligned}
\frac{\partial}{\partial t} g & =-2 \mathrm{Rc}+\frac{1}{2} \mathcal{H}-2 \nabla^{2} f, \\
\frac{\partial}{\partial t} T & \left.=\Delta_{L B} T-d(\nabla f\lrcorner T\right),
\end{aligned}
$$

where $f$ satisfies the conjugate heat equation

$$
\frac{\partial}{\partial t} f=-\Delta f-R+\frac{1}{4}|T|^{2} .
$$

For concreteness sake we now record the proof of Theorem 1.1.

Proof Clearly Equation (6-3) is diffeomorphism equivalent to (6-2). By combining Proposition 6.6 with Theorem 6.5, we obtain the statement of Theorem 1.1. 
Furthermore, Feldman, Ilmanen and Ni gave a generalization [11] of Perelman's steady and shrinking entropies to an entropy modeled on expanding solitons. Surprisingly, this expanding entropy has an extension to (6-2), as shown by the first named author. Define

$$
\begin{aligned}
\mathcal{W}_{+}(g, T, u, \sigma) & =\int_{M}\left[\sigma\left(\frac{|\nabla u|^{2}}{u}+R u-\frac{1}{12}|T|^{2} u\right)+u \log u\right] d V \\
& =\int_{M}\left[\sigma\left(\left|\nabla f_{+}\right|^{2}+R-\frac{1}{12}|T|^{2}\right)-f_{+}+n\right] u d V,
\end{aligned}
$$

where $f_{+}$is implicitly defined by $u=e^{-f_{+}} /(4 \pi \sigma)^{n / 2}$.

Theorem 6.7 [26, Theorem 6.2] Let $\left(M^{n}, g(t), T(t)\right)$ be a solution to (6-2) on $\left[t_{1}, t_{2}\right]$ and suppose $u(t)$ is the solution to (6-4). Let:

$$
v_{+}=\left[\left(t-t_{1}\right)\left(2 \Delta f_{+}-\left|\nabla f_{+}\right|^{2}+R-\frac{1}{12}|T|^{2}\right)-f_{+}+n\right] u
$$

Then:

$$
\begin{aligned}
\left(\frac{\partial}{\partial t}+\Delta\right. & \left.-R+\frac{1}{4}|T|^{2}\right) v_{+} \\
& \left.=\left.2\left(t-t_{1}\right)\left(\left|\mathrm{Rc}-\frac{1}{4} \mathcal{H}+\nabla^{2} f_{+}+\frac{g}{2 t}\right|^{2}+\frac{1}{4} \mid d^{*} T-\nabla f_{+}\right\lrcorner T\right|^{2}\right) u+\frac{1}{6}|T|^{2} u
\end{aligned}
$$

Corollary 6.8 Let $\left(M^{n}, g(t), T(t)\right)$ be a solution to (6-2) on $\left[t_{1}, t_{2}\right]$ and suppose $u(t)$ is a solution to the conjugate heat equation. Then:

$$
\begin{array}{rl}
\frac{\partial}{\partial t} \mathcal{W}_{+}\left(g(t), T(t), u(t), t-\tau_{1}\right)=\int_{M} & 2 u\left[\left(t-t_{1}\right)\left|\mathrm{Rc}-\frac{1}{4} \mathcal{H}+\nabla^{2} f_{+}+\frac{g}{2\left(t-t_{1}\right)}\right|^{2}\right. \\
+ & \left.\frac{1}{4}\left(t-t_{1}\right)\left|d^{*} T-\nabla f_{+}-T\right|^{2}+\frac{1}{12}|T|^{2}\right] d V
\end{array}
$$

We can derive further corollaries from these results, akin to the "ruling out of breathers" statements discovered by Perelman [22]. First recall two definitions.

Definition 6.9 We say that a solution to (6-2) is a breather if there are times $t_{1}<t_{2}$, a constant $\alpha>0$ and a diffeomorphism $\phi$ such that $\alpha g\left(t_{1}\right)=\phi^{*} g\left(t_{2}\right)$. The breather is steady, shrinking or expanding if $\alpha=1, \alpha<1$ or $\alpha>1$, respectively.

Definition 6.10 We say that a solution to (6-2) is a gradient soliton if there is a function $f$ and a constant $\lambda$ so that:

$$
\begin{aligned}
& 0=\mathrm{Rc}-\frac{1}{4} \mathcal{H}+\nabla^{2} f-\lambda g, \\
& 0=\Delta_{L B} T-d(\nabla f-T) .
\end{aligned}
$$


The soliton is steady, shrinking or expanding if $\lambda=0, \lambda>0$ or $\lambda<0$, respectively.

Corollary 6.11 Any solution to (6-2) that is a steady breather is a steady soliton. Any solution to (6-2) that is an expanding breather is an Einstein metric with $T \equiv 0$.

Proof The first statement follows immediately from Proposition 6.6. For the second, we note that Theorem 6.7 clearly implies that an expanding breather is an expanding soliton, and moreover $T \equiv 0$. Thus $g(t)$ is an expanding Ricci soliton, which are known to be negative constant Einstein metrics, a result originally due to Hamilton [15].

\section{Nonsingular solutions}

In this section we derive a strong topological consequence of the conjectural regularity picture of solutions to (1-1) by ruling out nonsingular solutions of (1-1) on Class $\mathrm{VII}^{+}$ surfaces.

Theorem 7.1 Suppose Conjecture 5.9 holds. Then any Class $\mathrm{VII}^{+}$surface contains an irreducible effective divisor of nonpositive self-intersection.

Proof We want to examine the volume-normalized version of (1-1). Let:

$$
\psi(\omega)=\frac{\int_{M} \operatorname{tr}_{\omega}\left[\partial \partial_{\omega}^{*} \omega+\bar{\partial} \bar{\partial}_{\omega}^{*} \omega+(\sqrt{-1} / 2) \partial \bar{\partial} \log \operatorname{det} g\right] d V}{\int_{M} d V}
$$

The volume normalized pluriclosed flow is:

$$
\begin{aligned}
\frac{\partial}{\partial t} \omega & =\partial \partial_{\omega}^{*} \omega+\bar{\partial} \bar{\partial}_{\omega}^{*} \omega+\frac{\sqrt{-1}}{2} \partial \bar{\partial} \log \operatorname{det} g-\frac{1}{n} \psi \omega, \\
\omega(0) & =\omega .
\end{aligned}
$$

Let $\left(M^{4}, J\right)$ be a complex surface of Class $\mathrm{VII}^{+}$. Note that by the theorem of Gauduchon [12], there are always pluriclosed metrics on complex surfaces, so we can find an initial condition for (7-1). If $M$ contains no irreducible divisor of nonpositive self-intersection, then Conjecture 5.9 automatically implies that the solution to (7-1) with any initial condition exists for all time with a uniform bound on curvature that moreover persists after scaling the diameter to unit size. Note that this implies the diameter is in fact bounded, for if not, by rescaling the diameter to unit size, we would produce a sequence of metrics with bounded curvature and volume approaching zero, which by Cheeger, Fukaya and Gromov [7], and Cheeger, Gromov and Taylor [8] would force $\chi(M)=0$, but $\chi(M)=b_{2}>0$. 
We want to derive a contradiction from the existence of such a flow. To do this we first identify the qualitative behavior of the corresponding solution to (1-1). Specifically, using monotone quantities we see that this solution exists for all time with volume growing quadratically. Thus to obtain the solution to (7-1) we must be uniformly scaling down this metric, and we will finish the proof by applying the expanding entropy formula. We begin with a definition and a series of lemmas.

Definition 7.2 Let $\left(M^{2 n}, g, J\right)$ be a Hermitian manifold. Let the degree of $(M, g)$ be

$$
d=\operatorname{deg}(M, g):=\int_{M}\left\langle c_{1}(M), \omega\right\rangle=\int_{M}\left(-\frac{\sqrt{-1}}{2} \partial \bar{\partial} \log \operatorname{det} g\right) \wedge \omega^{n-1} .
$$

More generally, given $\mathcal{L}$ a line bundle over $M$, define

$$
\operatorname{deg}(\mathcal{L}):=\int_{M} c_{1}(\mathcal{L}) \wedge \omega^{n-1}
$$

Note that the definition of degree is typically made with respect to a fixed Gauduchon metric, ie, a metric satisfying $\partial \bar{\partial} \omega^{n-1}=0$, so that the value does not depend on the representative of $c_{1}$. In the case $n=2$, Gauduchon metrics are the same as pluriclosed metrics, and the evolution of the degrees of line bundles is particularly clean.

Lemma 7.3 Let $\left(M^{4}, g(t), J\right)$ be a solution to (1-1) on a complex surface, and let $L$ be a line bundle over $M$. Then

$$
\frac{\partial}{\partial t} \operatorname{deg}_{g_{t}}(L)=-c_{1}(L) \cdot c_{1}(M)
$$

Lemma 7.4 Let $\left(M^{4}, g(t), J\right)$ be a solution to (1-1). Then the volume of $g(t)$ satisfies

$$
\frac{\partial}{\partial t} \operatorname{Vol}(g(t))=2 \int_{M}\left|\partial^{*} \omega\right|^{2}-d
$$

Next we would like to specialize these to the case of Class VII surfaces.

Lemma 7.5 Let $\left(M^{4}, g(t), J\right)$ be a solution to (1-1) on a Class VII surface with $b_{2}=n$. Then

$$
\operatorname{deg}_{g(t)}(M)=\operatorname{deg}_{g(0)}(M)+n t
$$

Proof This follows immediately from Lemma 7.3 and the fact that for Class VII surfaces, $c_{1}^{2}=-n$. 
Proposition 7.6 Let $\left(M^{4}, J\right)$ be a compact Class VII ${ }^{+}$surface. Suppose $\omega(t)$ is a solution to (7-1) on $M \times[0, \infty)$ with uniformly bounded curvature. Then the corresponding solution to (1-1) exists on $[0, \infty)$.

Proof Suppose the corresponding solution to unnormalized flow existed on $[0, \tau), \tau<$ $\infty$. First note that the degree of $M$ remains finite on $[0, \tau)$. However, to rescale to get the volume normalized flow we must be rescaling by a factor going to infinity since the curvature must be blowing up, and we have assumed the volume-normalized flow is nonsingular. Thus the volume must be going to zero. Using Lemma 7.4 we see that at some point the degree must be positive. But this condition is preserved, since the degree grows linearly by Lemma 7.5. In taking the rescaling limit, this says that the degree must go to infinity as $t \rightarrow \infty$ in the volume normalized flow. Since by assumption the volume normalized equation has bounded curvature, this is a contradiction.

Proposition 7.7 Let $\left(M^{4}, J\right)$ be a compact Class VII ${ }^{+}$surface. Suppose $\widetilde{\omega}(t)$ is a solution to (7-1) on $M \times[0, \infty)$ with uniformly bounded curvature. Then if $\omega(t)$ is the corresponding solution to (1-1), there exists a constant $C$ such that

$$
\frac{1}{C}\left(1+t^{2}\right) \leq \operatorname{Vol}(g(t)) \leq C\left(t^{2}+1\right) .
$$

Proof By assumption the corresponding solution to (7-1) has bounded curvature, and of course bounded volume. It follows that the scale-invariant quantity $d / \mathrm{Vol}^{1 / 2}$ is bounded along the solution to (7-1). Thus this quantity is bounded along (1-1) as well. By Lemma 7.5 it follows that

$$
d(0)+n t=d(t) \leq C \operatorname{Vol}(g(t))^{1 / 2}
$$

with $n>0$. The lower bound for $\operatorname{Vol}(g(t))$ follows by squaring the above inequality. Next we note the evolution equation for the degree under (7-1). In particular one has

$$
\frac{\partial}{\partial t} \operatorname{deg}(M)=-c_{1}^{2}+\operatorname{deg}(M)^{2}-2 \operatorname{deg} M \int_{M}\left|\partial^{*} \omega\right|^{2} .
$$

Since $\int_{M}\left|\partial^{*} \omega\right|^{2}$ is bounded, it easy follows that $\lim _{t \rightarrow \infty} \operatorname{deg}(M)>\epsilon>0$ for some $\epsilon>0$. It follows that the scale invariant quantity $\operatorname{deg}(M) / V^{1 / 2} \geq \epsilon>0$. Thus this inequality holds for the unnormalized flow as well, hence

$$
d(0)+n t=d(t) \geq \epsilon \operatorname{Vol}(g(t))^{1 / 2} .
$$

The upper volume bound now follows, completing the proof. 
We now give the proof of the theorem. From the proposition above we see that the solution to (7-1) is uniformly equivalent to a solution of

$$
\begin{aligned}
\frac{\partial}{\partial t} \omega & =\partial \partial_{\omega}^{*} \omega+\bar{\partial} \bar{\partial}_{\omega}^{*} \omega+\frac{\sqrt{-1}}{2} \partial \bar{\partial} \log \operatorname{det} g-\omega, \\
\omega(0) & =\omega .
\end{aligned}
$$

Moreover, this solution has uniformly bounded curvature and diameter. We claim that there exists a uniform lower bound on the injectivity radius as well. If there exists a sequence of points $\left(x_{i}, t_{i}\right)$ with $t_{i} \rightarrow \infty$ such that $\operatorname{inj}_{g_{t_{i}}}\left(x_{i}\right) \rightarrow 0$, then since there is a uniform diameter bound it follows from [8] that $\operatorname{inj}_{g_{t_{i}}}(x) \rightarrow 0$ for all points $x \in M$. In particular, the manifold $M$ admits a sequence of metrics collapsing with bounded curvature, which by [7] implies that $\chi(M)=0$. But for $\left(M^{4}, J\right)$ a complex Class VII $^{+}$surface one has $\chi(M)=b_{2}(M)>0$, so this is a contradiction, and so the lower injectivity radius bound follows.

Thus we can construct a blowdown limit for the unnormalized flow. Let $\lambda_{i} \rightarrow \infty$ and set

$$
g_{i}(t):=\frac{1}{\lambda_{i}} g\left(\lambda_{i} t\right)
$$

defined for $t>1 / \lambda_{i}$. By the estimates we have shown and the compactness theorem of [26], there is a subsequence of $\left\{\left(M, g_{i}(t), J\right)\right\}$, converging in the Cheeger-Gromov sense to a limiting pluriclosed flow $\left\{\left(M_{\infty}, g_{\infty}(t), J_{\infty}\right)\right\}$. Since $\mathcal{W}_{+}$is invariant under the blowdown rescaling, and is monotone increasing and bounded above, it is clear that $W_{+}$is constant along $g_{\infty}$, which thus must be an expanding soliton, and hence is a Kähler-Einstein metric. In particular, we have that $\left(M_{\infty}, g_{\infty}(1), J\right)$ is a Kähler manifold. By the Hodge decomposition, since $\left(M_{\infty}, J_{\infty}\right)$ is Kähler we have that $b_{1}\left(M_{\infty}\right)$ is even. On the other hand, by the diameter and injectivity radius bounds, we have that $M \cong M_{\infty}$ and $b_{1}(M)=1$. This is a contradiction, finishing the proof.

By general theory [20, Lemma 2.2] the curve is either a rational curve, rational curve with double point or an elliptic curve. If the curve is elliptic, the manifold is known (Nakamura [20], and Enoki [10]). Furthermore, Class VII ${ }^{+}$surfaces that contain $b_{2}$ rational curves automatically contain a global spherical shell by the result of Dloussky, Oeljeklaus and Toma [9]. Therefore we see that Conjecture 5.9 implies the classification of Class $\mathrm{VII}^{+}$surfaces with $b_{2}=1$, a theorem obtained by Teleman using gauge theory [29]. Furthermore it implies a concrete complex analytic conclusion on any Class $\mathrm{VII}^{+}$ surface. It seems likely that a more detailed analysis of the limit points can yield the entire classification of Class $\mathrm{VII}^{+}$surfaces as a consequence of Conjecture 5.9. 


\section{Conclusion}

Given the results contained herein, Equation (1-1) clearly seems to be a very natural parabolic equation on complex manifolds. By the results of [27], the corresponding elliptic (static) equation, ie,

$$
P^{1,1}=\lambda \omega,
$$

seems very closely related to the Kähler-Einstein condition, and we further seen here the relationship of solutions to (1-1) and the topological and complex structure of surfaces. While there are only a few large classes of examples of complex manifolds of dimension $n \geq 3$ admitting pluriclosed (but not Kähler) geometries, it seems likely that understanding the existence problem for static metrics in higher dimensions will have relevance. We take the time here to observe some further structural results for static metrics in any dimension. First of all, we recall the Bochner formula for holomorphic forms on complex manifolds.

Theorem 8.1 (Kobayashi and Wu [17], and Bochner [3]) Let $\left(M^{2 n}, g, J\right)$ be a Hermitian manifold. Fix $\eta$ a holomorphic $(p, 0)-$ form. Then

$$
\Delta|\eta|^{2}=|\nabla \eta|^{2}+|\bar{\nabla} \eta|^{2}+\langle S \circ \eta, \eta\rangle .
$$

Here, $\Delta=\operatorname{tr}_{\omega} \partial \bar{\partial}$ is the canonical Laplacian and $\nabla$ is the Chern connection. Also, $S \circ \eta$ is the natural action induced on $\Lambda^{p, 0}$ of an endomorphism of $T^{1,0}$. In particular, in coordinates:

$$
(S \circ \eta)_{i_{1} \cdots i_{p}}=\frac{1}{p !} \sum_{j=1}^{p} S_{i_{j}}^{k} \eta_{i_{1} \cdots i_{j-1} k i_{j+1} \cdots i_{p}}
$$

Corollary 8.2 [16, Corollary 4.4]

- Let $\left(M^{2 n}, \omega, J\right)$ be a compact complex manifold with $\pi c_{1}=0$. Suppose $\omega$ is a static metric, which necessarily has $s \equiv 0$. Then every holomorphic $(p, 0)$-form is parallel with respect to the Chern connection.

- Let $\left(M^{2 n}, \omega, J\right)$ be a compact complex manifold with $\pi c_{1}>0$. Suppose $\omega$ is a static metric, which necessarily has $s \equiv c>0$. Then $H^{0}\left(M, \Lambda^{p}\right)=0$, $p=1, \ldots, n$.

Note that in the second part of the above corollary, we mean $c_{1}>0$ as a class in the Aeppli cohomology group $\mathcal{H}_{\partial+\bar{\partial}}^{1,1}$ as defined in Section 5 . These are precisely the corollaries to his conjecture observed by Calabi [6] in the Kähler setting, indicating that (1-1) is a very natural extension of the Calabi-Yau/Kähler Ricci flow theory. We close with a final vanishing result for static metrics: 
Proposition 8.3 Let $\left(M^{2 n}, g, J\right)$ be a complex manifold with either $c_{1}=0$ or $c_{1}>0$, with $g$ a static metric. Then either $g$ is Kähler or $h^{n-1,0}=0$.

Proof Since $Q^{1} \geq 0$, and $\lambda \geq 0$ by the assumption on the first Chern class, (8-1) clearly implies that $S \geq Q^{1}$. Therefore by applying the maximum principle to (8-2) we conclude that every holomorphic section $\eta$ of $\Lambda^{n-1,0}$ is parallel with respect to the Chern connection. In particular, it is of constant norm. If $g$ is not Kähler, there is a point $p \in M$ where the torsion tensor does not vanish identically. Specifically, we can pick complex coordinates where $S$, and hence $Q^{1}$, are diagonalized. Without loss of generality $T_{12 \bar{j}} \neq 0$. Thus

$$
Q_{1 \overline{1}}^{1}>0, \quad Q_{2 \overline{2}}^{1}>0
$$

(see Lemma 2.1 for the expression of $\left.Q^{1}\right)$. It follows that $S(p) \geq Q^{1}(p)$ is $n-1$ positive at $p$. By the form of the Bochner formula (8-2), if $\eta$ does not vanish we conclude that $\Delta|\eta|^{2}(p)>0$, contradicting that $\eta$ is parallel.

\section{References}

[1] B Alexandrov, S Ivanov, Vanishing theorems on Hermitian manifolds, Differential Geom. Appl. 14 (2001) 251-265 MR1836272

[2] W P Barth, K Hulek, C A M Peters, A Van de Ven, Compact complex surfaces, 2nd edition, Ergeb. Math. Grenzgeb. 4, Springer, Berlin (2004) MR2030225

[3] S Bochner, Vector fields and Ricci curvature, Bull. Amer. Math. Soc. 52 (1946) 776797 MR0018022

[4] N Buchdahl, On compact Kähler surfaces, Ann. Inst. Fourier (Grenoble) 49 (1999) 287-302 MR1688136

[5] N Buchdahl, A Nakai-Moishezon criterion for non-Kähler surfaces, Ann. Inst. Fourier (Grenoble) 50 (2000) 1533-1538 MR1800126

[6] E Calabi, On Kähler manifolds with vanishing canonical class, from: "Algebraic geometry and topology. A symposium in honor of S Lefschetz", Princeton Univ. Press (1957) 78-89 MR0085583

[7] J Cheeger, K Fukaya, M Gromov, Nilpotent structures and invariant metrics on collapsed manifolds, J. Amer. Math. Soc. 5 (1992) 327-372 MR1126118

[8] J Cheeger, M Gromov, M Taylor, Finite propagation speed, kernel estimates for functions of the Laplace operator, and the geometry of complete Riemannian manifolds, J. Differential Geom. 17 (1982) 15-53 MR658471

[9] G Dloussky, K Oeljeklaus, M Toma, Class VII $_{0}$ surfaces with $b_{2}$ curves, Tohoku Math. J. 55 (2003) 283-309 MR1979500 
[10] I Enoki, On surfaces of class $\mathrm{VII}_{0}$ with curves, Proc. Japan Acad. Ser. A Math. Sci. 56 (1980) 275-279 MR581470

[11] M Feldman, T Ilmanen, L Ni, Entropy and reduced distance for Ricci expanders, J. Geom. Anal. 15 (2005) 49-62 MR2132265

[12] P Gauduchon, Le théorème de l'excentricité nulle, C. R. Acad. Sci. Paris Sér. A-B 285 (1977) A387-A390 MR0470920

[13] B Guan, Q Li, Complex Monge-Ampere equations on Hermitian manifolds arXiv: 0906.3548

[14] R S Hamilton, Three-manifolds with positive Ricci curvature, J. Differential Geom. 17 (1982) 255-306 MR664497

[15] R S Hamilton, Non-singular solutions of the Ricci flow on three-manifolds, Comm. Anal. Geom. 7 (1999) 695-729 MR1714939

[16] S Ivanov, G Papadopoulos, Vanishing theorems and string backgrounds, Classical Quantum Gravity 18 (2001) 1089-1110 MR1822270

[17] S Kobayashi, H-H Wu, On holomorphic sections of certain Hermitian vector bundles, Math. Ann. 189 (1970) 1-4 MR0270392

[18] A Lamari, Le cône kählérien d'une surface, J. Math. Pures Appl. 78 (1999) 249-263 MR1687094

[19] G M Lieberman, Second order parabolic differential equations, World Scientific Publishing Co., River Edge, NJ (1996) MR1465184

[20] I Nakamura, On surfaces of class $\mathrm{VII}_{0}$ with curves, Invent. Math. 78 (1984) 393-443 MR768987

[21] T Oliynyk, V Suneeta, E Woolgar, A gradient flow for worldsheet nonlinear sigma models, Nuclear Phys. B 739 (2006) 441-458 MR2214659

[22] G Perelman, On the entropy formula for Ricci flow and its geometric applications arXiv:math/0211159

[23] D H Phong, N Sesum, J Sturm, Multiplier ideal sheaves and the Kähler-Ricci flow, Comm. Anal. Geom. 15 (2007) 613-632 MR2379807

[24] J Polchinski, String theory, I: an introduction to the bosonic string, Cambridge Monographs on Mathematical Physics, Cambridge Univ. Press (1998) MR1648555

[25] N Šešum, Curvature tensor under the Ricci flow, Amer. J. Math. 127 (2005) 1315-1324 MR2183526

[26] J Streets, Regularity and expanding entropy for connection Ricci flow, J. Geom. Phys. 58 (2008) 900-912 MR2426247

[27] J Streets, G Tian, A parabolic flow of pluriclosed metrics, Int. Math. Res. Not. 2010 (2010) 3101-3133 MR2673720 
[28] J Streets, G Tian, Hermitian curvature flow, J. Eur. Math. Soc. 13 (2011) 601-634 MR2781927

[29] A Teleman, Donaldson theory on non-Kählerian surfaces and class VII surfaces with $b_{2}=1$, Invent. Math. 162 (2005) 493-521 MR2198220

[30] A Teleman, The pseudo-effective cone of a non-Kählerian surface and applications, Math. Ann. 335 (2006) 965-989 MR2232025

[31] G Tian, New results and problems on Kähler-Ricci flow, from: "Géométrie différentielle, physique mathématique, mathématiques et société, II”, Astérisque 322, Soc. Math. France (2008) 71-92 MR2521654

[32] G Tian, Z Zhang, On the Kähler-Ricci flow on projective manifolds of general type, Chinese Ann. Math. Ser. B 27 (2006) 179-192 MR2243679

[33] B Weinkove, The Calabi-Yau equation on almost-Kähler four-manifolds, J. Differential Geom. 76 (2007) 317-349 MR2330417

Department of Mathematics, University of California, Irvine Rowland Hall, Irvine, CA 92617, USA

Department of Mathematics, Princeton University

Fine Hall, Princeton, NJ 08544, USA

jstreets@uci.edu, tian@math.princeton.edu

http://www-math.mit.edu/ tian/

Proposed: Tom Mrowka

Received: 1 March 2013

Seconded: Tobias Colding, Ronald Stern

Accepted: 29 May 2013 